\title{
An omni-directional multirotor vehicle
}

Journal Article

Author(s):

Brescianini, Dario; D'Andrea, Raffaello

Publication date:

2018-11

Permanent link:

https://doi.org/10.3929/ethz-b-000295701

Rights / license:

In Copyright - Non-Commercial Use Permitted

Originally published in:

Mechatronics 55, https://doi.org/10.1016/j.mechatronics.2018.08.005 


\title{
An Omni-Directional Multirotor Vehicle
}

\author{
Dario Brescianini and Raffaello D'Andrea
}

\begin{abstract}
In this paper we present the design, modelling and control of an omni-directional multirotor vehicle, i.e. a fullyactuated vehicle that can hover at any attitude and accelerate in any direction. Based on a static force and torque analysis for generic rotor configurations, an octorotor configuration is derived that maximizes the vehicle's agility while rendering its characteristics almost rotationally invariant. A prototype vehicle with the derived rotor configuration is built using reversible fixed-pitch rotors that can generate positive and negative thrust, enabling the vehicle to independently control its thrust and torque in all three dimensions. A control scheme that allows for simultaneously tracking a desired position and attitude trajectory is introduced. Experimental results demonstrate the feasibility of the vehicle design and control strategy.
\end{abstract}

Index Terms-Aerial robotics, unmanned aerial vehicles, mechanics, design and control.

\section{INTRODUCTION}

Unmanned aerial vehicles have seen a boost in popularity over the past decade and are now increasingly applied commercially across various industrial sectors, ranging from agriculture to filmmaking and entertainment [1]. Often, multirotor vehicles such as quadrocopters or hexacopters are employed in these applications due to their agility, hover-capability and mechanical simplicity. However, these multirotor vehicles are under-actuated [2], i.e. unable to control all of their six degrees-of-freedom independently. In order to maximize performance criteria such as flight duration, range or payload, the rotors of multirotor vehicles are typically arranged in a single plane, which limits the thrust the vehicles can generate to a single direction normal to their rotors. As a result, the vehicles' position and attitude dynamics are coupled and in order to accelerate in a desired direction, the vehicles have to rotate such that their rotor disk normal is aligned with the desired direction of acceleration.

\section{A. Goal and Motivation}

The inability of traditional multirotor vehicles to generate thrust and torque independently of each other and in any direction has far reaching consequences: Firstly, the vehicles' sets of feasible flight maneuvers are severely limited due to the coupling of their position and attitude dynamics. Secondly, the vehicles are unable to resist arbitrary force and torque disturbances without a substantial delay. This is due to the time required to reorient the vehicles' body-fixed thrust direction and degrades the vehicles' performance in scenarios that demand high precision flight [3] and in scenarios where large external disturbances are encountered, as in the emerging field of aerial physical interaction (for example grasping and

The authors are members of the Institute for Dynamic Systems and Control, ETH Zurich, Switzerland. \{bdario, rdandrea\} @ethz . ch

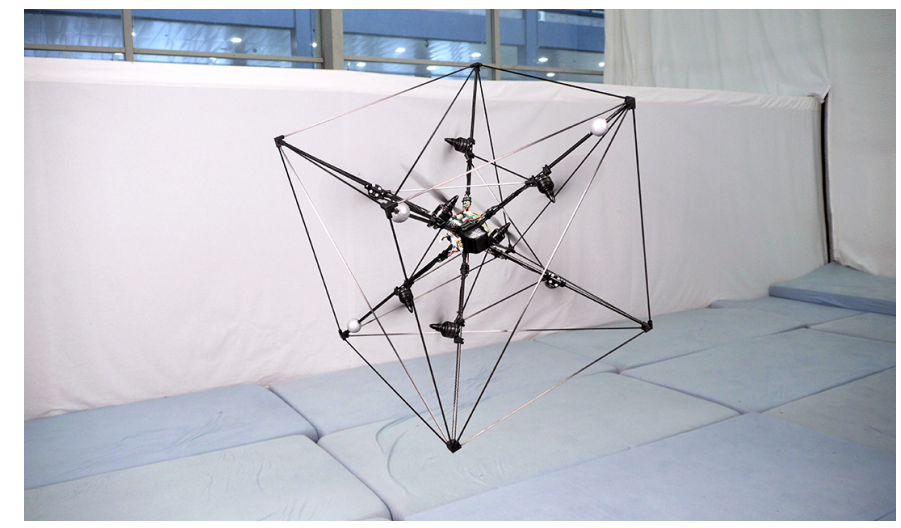

Fig. 1: The omni-directional multirotor hovering at an arbitrary attitude.

object manipulation [4], [5], aerial construction [6], or human interaction [7], [8]).

This article aims to overcome the limitations of traditional multirotor vehicles by developing a fully-actuated multirotor vehicle, i.e. a vehicle capable of independently controlling its thrust and torque in all three dimensions. In particular, the objective is to develop an omni-directional multirotor vehicle that is able to hover at any attitude and accelerate in any direction (see Fig. 1). Due to its ability to generate thrust and torque in any direction, such a multirotor vehicle will render novel flight maneuvers possible, which can, for example, be exploited by applications that require an unconstrained motion range such as aerial filming or manipulation. In these applications, the constrained motion range of traditional multirotor vehicles is often made up for by a multi-degrees-of-freedom robot arm attached to the vehicle, which yields systems that are very complex to handle [9], [10]. Furthermore, although fullyactuated multirotor vehicles are also unable to resist arbitrary force and torque disturbances without any delay, their total thrust and torque dynamics are in general significantly faster than the attitude dynamics of traditional multirotor vehicles [3], rendering them favourable for aerial physical interaction tasks.

\section{B. Related Work}

Several fully-actuated multirotor vehicle designs that allow control of all of the six degrees-of-freedom independently have been developed in recent years and can be roughly divided into two categories.

The first category comprises vehicles with non-planar rotor configurations where the orientations of the rotor disks with respect to the vehicle are fixed. By adjusting the amount of thrust each individual rotor generates, these vehicles are able to control the direction of their total thrust and torque. 
In order to possess full thrust and torque authority in all three dimensions, at least six rotors arranged on at least three different planes are required. In [11]-[13], hexrotor vehicle designs are studied in which the rotors are spaced evenly around the circumference of a circle and pairwise tilted about their radial axis, such that the rotor disk normals span the three dimensional Euclidian space. A similar approach is pursued in [14], but the rotors are additionally tilted about their tangential axis, allowing for more design flexibility. In [15] and [16], multirotor vehicle designs are presented where both the positions of the rotors and their orientations are determined by solving an optimization problem. The objective of [15] is to minimize the vehicle volume while maintaining a certain thrust and torque controllability, whereas the objective of [16] is to maximize the smallest maximum thrust and torque that can be generated in any direction.

The benefit of full thrust and torque authority of non-planar rotor configurations comes at the expense of reduced energy efficiency with respect to planar rotor configurations due to higher internal forces. This negative side effect is reduced by vehicles of the second category, which can adjust the orientation of their rotors during flight and thereby control the direction of their total thrust and torque. In [17] and [18], quadrotor vehicle designs are studied which use servomotors to change the alignment of the rotors. In [19], a rotor configuration consisting of three small variable-orientation rotors mounted horizontally around two large counter-rotating coaxial rotors is proposed. The two large rotors are responsible for efficiently generating enough lift to overcome gravity, while the small rotors are used to provide lateral forces.

The advantage of increased efficiency of variable-orientation rotor configurations comes at the expense of significantly more mechanical complexity, weight and cost. In addition, even though tilting the rotors solves the problem of underactuation and allows the vehicle to independently generate thrust and torque in any direction, the thrust and torque response may not be fast enough to effectively resist arbitrary force and torque disturbances due to the time required to adjust the orientation of the rotor disks. In [20], an attempt is made to combine the advantages of both categories with a vehicle design consisting of six tilting rotors arranged on three different planes. The proposed vehicle design allows for fast changes of the vehicle's thrust and torque by only adjusting the thrust produced by the individual rotors, and tilting the rotors is used to modify the rotor configuration from energy efficient configurations with little control authority (almost planar rotor configurations) to configurations with a lot of control authority but less efficiency.

\section{Contribution}

Although several multirotor vehicle designs exist that are capable of independently generating thrust and torque in any direction, they are typically designed with a preferred direction of orientation in which their particular arrangement of rotors makes them most effective in overcoming gravity. As a result, these multirotor vehicles are typically not able to hover at arbitrary attitudes due to rotor thrust constraints, even though they are fully-actuated. This paper presents the design, modelling and control of an omni-directional multirotor vehicle, i.e. a fully-actuated multirotor vehicle that can provide sufficient thrust and torque in any direction in order to hover at any attitude and accelerate in any direction.

The vehicle design is formulated as an optimization problem based on a static thrust and torque analysis for generic rotor configurations. The objective is to find the rotor configuration that maximizes the vehicle's agility, while rendering its characteristics as rotationally invariant as possible. The outcome of the optimization is an octorotor vehicle design with fixed rotor disk orientations, where the rotors are pairwise aligned on four different planes. By using rotors capable of generating positive and negative thrust, the vehicle is able to independently generate a total thrust and torque in any direction, which decouples its position and attitude dynamics and enables it to fly novel maneuvers.

A control strategy is developed that allows for simultaneously tracking a desired position and attitude trajectory and thereby taking full advantage of the vehicle's omnidirectionality and decoupled position and attitude dynamics. The control strategy is based on multiple cascaded control loops, where it is assumed that the inner control loops can track the input commands arbitrarily fast. Using loop shaping and feedback linearization, each control loop is designed to respond to input commands in the fashion of a linear first or second-order system. All control gains can thus be expressed in terms of time constants and damping ratios, allowing for intuitive tuning of the control loops. Since the vehicle is overactuated (it has eight rotors to control six degrees-of-freedom), a control allocation strategy is introduced with the objective of minimizing the vehicle's power consumption while taking the rotors' thrust constraints and dynamics into account.

A prototype vehicle with the proposed octorotor configuration is built and used to experimentally evaluate the performance of the vehicle design and control strategy. Different flight maneuvers are executed to assess the vehicle's omnidirectionality and ability to simultaneously track decoupled position and attitude maneuvers. Futher experiments are conducted to identify the effects of aerodynamic interference between rotors that are neglected during the design of the vehicle and controllers.

Preliminary results on the design and control of the vehicle were presented in a conference paper [21]. In addition to providing a more thorough description of the vehicle design and implementation, this paper extends the previously published results

- by computing the optimal rotor configuration for different numbers of rotors,

- by providing an analysis of the attainable set of thrusts and torques,

- by introducing a control allocation strategy taking the rotor dynamics and the set of attainable rotor thrusts into account,

- and by experimentally identifying aerodynamic interference between the rotors. 


\section{Outline}

The remainder of this paper is organized as follows: Section II presents the design of an omni-directional multirotor vehicle. Section III describes the implementation of a prototype vehicle based on the derived vehicle design. Section IV introduces a model of the vehicle's dynamics, and in Section $\mathrm{V}$, a cascaded control scheme for the independent control of the vehicle's position and attitude is devised. In Section VI, the feasibility of the vehicle design and control strategy is demonstrated through experimental flight tests. Concluding remarks are made in Section VII.

\section{VEHICLE DESIGN}

This section describes the design of an omni-directional multirotor vehicle. A multirotor vehicle's properties are mainly determined by its rotor configuration, i.e. the number of rotors, their position and their orientation. The objective of the vehicle design is to find a rotor configuration that maximizes the vehicle's agility while rendering its characteristics as rotationally invariant as possible. For ease of notation, vectors may be expressed as $n$-tuples $\boldsymbol{x}=\left(x_{1}, x_{2}, \ldots, x_{n}\right)$ with dimensions and stacking clear from context. Unless otherwise stated, all three dimensional vectors are expressed with respect to a bodyfixed coordinate frame $\mathcal{B}$ with its origin at the vehicle's center of mass.

\section{A. Design Considerations}

A wide class of multirotor vehicles exists which differ in their ability to adjust the orientation of their rotor disks and in how the rotors generate lift. Before starting with the vehicle design, the type of multirotor vehicle to be considered is specified.

1) Variable-orientation vs. fixed-orientation rotor disks: Multirotor vehicles capable of adjusting the orientation of their rotor disks have the advantage that they can align the rotor disks such that the desired total thrust and torque are generated efficiently (see, for example, [20] and references therein). However, the gain in efficiency comes at the expense of additional mechanical complexity and weight compared to a vehicle design with fixed rotor disk orientations due to the additional actuators required to adjust the rotor disk orientations. Furthermore, if the number of rotors is less than six, variable-orientation rotor configurations generally require the reorientation of their rotor disks in order to change the total thrust and torque, which is considered to be slower than changing the total thrust and torque by adjusting the individual rotor thrusts and also more complex to control due to gyroscopic torques acting on the vehicle when changing the orientation of the rotors [12]. For these reasons, the vehicle design is limited to rotor configurations with fixed rotor disk orientations.

2) Variable-pitch vs. fixed-pitch rotors: In order for a multirotor vehicle to be omni-directional, i.e. to be able to generate sufficient thrust to hover at any attitude and accelerate in any direction, it is beneficial if the rotors can produce both positive and negative thrust, such that the load can be distributed more evenly among all rotors. Generating both positive and negative thrust can be accomplished by either using variable-pitch rotors and changing the rotor's angle of attack from positive to negative or by using fixed-pitch rotors and reversing the direction of rotation. In [22] it was demonstrated that variable-pitch rotors allow for faster thrust changes than fixed-pitch rotors, in particular when changing from a positive to a negative thrust or vice versa. However, variable-pitch rotors require an additional actuator to set the pitch angle and are mechanically more complex due to the many moving parts. Therefore, due to weight considerations and their mechanical simplicity, only fixed-pitch rotors are considered in the vehicle design.

The thrust generated by a fixed-pitch rotor can be changed by changing the rotor's angular velocity. Because most brushless electronic speed controllers (ESCs) rely on measuring the motor's back electromotive force to estimate its position and control the commutations, a minimum angular velocity and hence minimum thrust is required in order for the motor to function properly. The thrust magnitude of a fixed-pitch rotor is thus constrained to

$$
0<f_{\min } \leq\left|f_{\text {rot }}\right| \leq f_{\max }
$$

where the upper thrust constraint can be due to many constraints, such as the maximum voltage supplied by the battery that can be applied to the motor or the motor's heat dissipation capacity.

\section{B. Static Thrust and Torque Analysis}

Consider a generic $N$-rotor configuration where the $i$-th rotor produces a thrust of magnitude $f_{\text {rot }, i}$ along the fixed rotor disk normal $\boldsymbol{n}_{i}$. Then, the total thrust generated by all $N$ rotors is given by

$$
\boldsymbol{f}=\sum_{i=1}^{N} f_{\mathrm{rot}, i} \boldsymbol{n}_{i}
$$

Each rotor thrust $f_{\text {rot, } i}$ causes a torque about the vehicle's center of mass due to the off-center mounting of the rotors, and additionally due to aerodynamic drag, a torque that acts in the opposite direction to the rotor's direction of rotation. The total torque acting on the vehicle can therefore be summarized as

$$
\boldsymbol{t}=\sum_{i=1}^{N} f_{\mathrm{rot}, i}\left(\boldsymbol{p}_{i} \times \boldsymbol{n}_{i}\right)+\kappa_{i} f_{\mathrm{rot}, i} \boldsymbol{n}_{i},
$$

where $\boldsymbol{p}_{i}$ is the position of the $i$-th rotor relative to the vehicle's center of mass, the symbol $\times$ denotes the cross product and $\kappa_{i}$ is the rotor specific thrust-to-drag ratio that also encodes the rotor's direction of rotation (see Section IV-A for details). The torque due to the aerodynamic drag of the rotors is typically an order of magnitude smaller than the torque due to the off-center mounting of the rotors ${ }^{1}$ and is thus assumed to be negligible for the envisioned vehicle design, where torque

\footnotetext{
${ }^{1}$ This is based on the assumption that the rotors are placed at a distance of at least a rotor diameter away from the vehicle's center of mass [23].
} 
can be generated in any direction by exploiting the rotors' offcenter mounting. The thrust and torque expressions (2) and (3) can then be summarised as

$$
\left[\begin{array}{l}
f \\
t
\end{array}\right]=\underbrace{\left[\begin{array}{c}
N \\
P \times N
\end{array}\right]}_{=: \tilde{B}} f_{\text {rot }},
$$

with $\boldsymbol{N}$ and $\boldsymbol{P}$ being $3 \times N$ matrices composed of the column vectors $\boldsymbol{n}_{i}$ and $\boldsymbol{p}_{i}$, respectively, and with the $i$-th column of $\boldsymbol{P} \times \boldsymbol{N}$ being defined as $\boldsymbol{p}_{i} \times \boldsymbol{n}_{i}$.

For the sake of tractability of the vehicle design problem, it is further assumed that the minimum rotor thrust $f_{\min }$ is zero, since the minimum thrust magnitude is very small in practice and because it greatly simplifies the computation of the set of attainable thrusts and torques ${ }^{2}$. The simplified set of attainable thrusts and torques, denoted by $\tilde{\mathcal{V}}$, is given by

$$
\tilde{\mathcal{V}}=\left\{\tilde{\boldsymbol{B}} \boldsymbol{f}_{\text {rot }} \in \mathbb{R}^{6} \mid \boldsymbol{f}_{\text {rot }} \in \mathbb{R}^{N},\left\|\boldsymbol{f}_{\text {rot }}\right\|_{\infty} \leq f_{\text {max }}\right\},
$$

where $\tilde{B}$ is the matrix defined in (4) that maps the individual rotor thrusts $f_{\text {rot }}$ to total thrust and torque $v=(f, t)$, i.e. $\boldsymbol{v}=\tilde{\boldsymbol{B}} \boldsymbol{f}_{\text {rot }}$.

\section{Rotor Configuration Optimization}

We seek to find the rotor configuration that maximizes the vehicle's agility while rendering its characteristics as rotationally invariant as possible. Since every additional rotor adds mass and complexity to the vehicle, we look for the rotor configuration with the minimum number of rotors. In the following, the vehicle design is formulated as an optimization problem based on the static thrust and torque analysis.

1) Design Objective: The objective of the vehicle design is to find the rotor configuration that maximizes the vehicle's agility, where the smallest Euclidean norm of the maximum attainable thrust and torque over all directions is taken as a measure of the vehicle's agility. Expressed differently, we seek to find the rotor configuration such that the radius of the largest Euclidean ball centered at the origin that is fully enclosed by the attainable thrust and torque set $\tilde{\mathcal{V}}$ is maximized.

2) Design Constraints: In order for the multirotor vehicle to be rotationally invariant, it must fulfill two requirements: Firstly, it must be able to generate an equal amount of thrust and torque in any direction, and secondly, its moment of inertia must be rotationally invariant.

The first requirement cannot be achieved with a finite number of rotors, however, the design objective can be seen as an approximation thereof as it guarantees that an equal minimum amount of thrust and torque can be generated in any direction. To further enforce rotational invariance, we also demand that the maximum amount of thrust and torque that can be generated in the different directions are of equal magnitude, i.e. that all vertices of the set of attainable thrusts and torques are equally distant from the origin. These extrema are achieved when the individual rotors generate thrusts of magnitude $\left|f_{\text {rot }, i}\right|=f_{\max }$, i.e. when $\left\|\boldsymbol{f}_{\text {rot }}\right\|_{2}=\sqrt{N} f_{\max }$. By requiring that the all singular values of the thrust and torque

\footnotetext{
${ }^{2} \mathrm{~A}$ non-zero minimum rotor thrust $f_{\min }$ and its implications on the vehicle design will be considered in a later step.
}

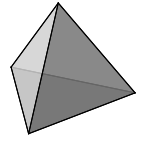

(a) $N=4$

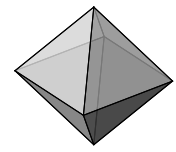

(b) $N=6$

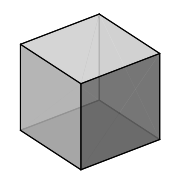

(c) $N=8$

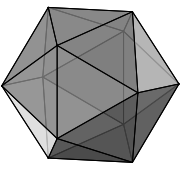

(d) $N=12$

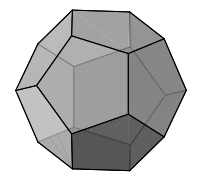

(e) $N=20$
Fig. 2: If the rotors are constrained to the vertices of a regular solids, i.e. a tetrahedron (a), an octahedron (b), a cube (c), an icosahedron (d), a dodecahedron (e) or combinations of regular solids with coinciding centers, then the vehicle's moment of inertia is rotationally invariant.

map $\tilde{\boldsymbol{B}}$ are equal, or equivalently that the smallest and largest singular values are equal,

$$
\sigma_{\max }(\tilde{\boldsymbol{B}})=\sigma_{\min }(\tilde{\boldsymbol{B}}),
$$

it is ensured that for a given amount rotor thrusts $\left\|f_{\text {rot }}\right\|_{2}$, an equal amount of thrust and torque $\left\|\tilde{\boldsymbol{B}} \boldsymbol{f}_{\text {rot }}\right\|_{2}$ can be generated in any direction, i.e.

$$
\frac{\left\|\tilde{\boldsymbol{B}} \boldsymbol{f}_{\text {rot }}\right\|_{2}}{\left\|\boldsymbol{f}_{\mathrm{rot}}\right\|_{2}}=\text { const }, \quad \forall \boldsymbol{f}_{\text {rot }} \in\left\{\boldsymbol{y} \in \mathbb{R}^{N} \mid \boldsymbol{y} \perp \operatorname{Null}(\tilde{\boldsymbol{B}})\right\} .
$$

Note that in (7) it is necessary that the rotor thrusts do not lie in the null space of $\tilde{\boldsymbol{B}}$ as these rotor thrusts do not contribute to the total thrust and torque.

Let $\boldsymbol{J}$ denote the vehicle's moment of inertia with respect to its center of mass expressed in the vehicle's body-fixed frame, and let $\boldsymbol{J}^{\prime}$ be the moment of inertia described in a frame rotated by any rotation matrix $\boldsymbol{R} \in S O(3)$ with respect to the body frame. $\boldsymbol{J}^{\prime}$ is then given by

$$
\boldsymbol{J}^{\prime}=\boldsymbol{R} \boldsymbol{J} \boldsymbol{R}^{T}
$$

In order for the moment of inertia to be rotationally invariant, it must hold that $\boldsymbol{J}^{\prime}=\boldsymbol{J}$ for any $\boldsymbol{R} \in S O(3)$, which implies that $\boldsymbol{J} \boldsymbol{R}=\boldsymbol{R} \boldsymbol{J}$ and consequently that the inertia tensor is a multiple of the identity matrix, i.e. that all principle moments of inertia are equal. In [24], it is shown that the moment of inertia only reduces to a multiple of the identity matrix for solids that have at least two $n$-fold rotational axes (with $n \geq 3$ ) such as regular solids. If it is assumed that the vehicle's moment of inertia is mainly determined by its rotor positions and that the rotors can be approximated by point masses, then the rotors have to lie on the vertices of regular solids (see Fig. 2) or combinations of regular solids with coinciding centers. Since at least six rotors are required in order for the vehicle to be able to independently control its thrust and torque in any direction, or equivalently for $\tilde{\boldsymbol{B}}$ to have full rank, the set of 
possible rotor positions $\mathcal{P}$ is constrained to regular solids and combinations thereof with at least six vertices.

Note that all vertices of regular solids are equidistant from the center. In order for the vehicle design to be scale invariant, the rotors were chosen to lie on the unit sphere, i.e. $\left\|\boldsymbol{p}_{i}\right\|_{2}=1$, such that the torques are normalized and a rotor thrust of one unit results in at most one unit of total thrust and one unit of total torque.

3) Optimization Problem: The vehicle design problem can formally be stated as follows: We seek to find the rotor configuration, i.e. the rotor positions $\boldsymbol{P}$ and rotor disk normals $N$, that solves

$$
\begin{array}{ll}
\underset{\boldsymbol{P}, \boldsymbol{N}}{\operatorname{maximize}} & \arg \max _{r}\left\{r:\left\{\boldsymbol{v} \in \mathbb{R}^{6} \mid\|\boldsymbol{v}\|_{2} \leq r\right\} \subseteq \tilde{\mathcal{V}}\right\} \\
\text { subject to } & \left\|\boldsymbol{n}_{i}\right\|_{2}=1, \quad \forall i \in\{1, \ldots, N\}, \\
& \boldsymbol{P} \in \mathcal{P}, \\
& \sigma_{\max }(\tilde{\boldsymbol{B}})=\sigma_{\min }(\tilde{\boldsymbol{B}}) .
\end{array}
$$

4) Discussion: Note that in the design objective of (9), the total thrust $\boldsymbol{f}$ and the total torque $t$ are equally weighted, but in practice, one may be more important than the other. However, it was numerically found that the optimal rotor configuration, i.e. the rotor disk normals $\boldsymbol{N}$ and the rotor positions $\boldsymbol{P}$ (up to scale), are independent of the weighting of the total thrust $f$ and the total torque $t$ used in the optimization problem, or equivalently of the size of $\left\|\boldsymbol{p}_{i}\right\|_{2}$. The distance of the rotors from the vehicle's center of mass can therefore be chosen in a later step according to the torque requirements. The longer the distance of the rotors from the vehicle's center of mass, the more torque can be generated (without decreasing the total thrust). However, because the torque scales linearly with the distance while the vehicle's inertia scales quadractically with the distance, a tradeoff between the vehicle's maximum torque and agility has to be made [2].

\section{Optimal Rotor Configuration}

The constrained nonlinear optimization problem (9) was solved numerically using MATLAB's fmincon-routine, and Fig. $3 \mathrm{a}-3 \mathrm{~d}$ show the optimal rotor configurations for the rotor positions constrained to an octahedron $(N=6)$, a cube $(N=8)$, an icosahedron $(N=12)$ and a dodecahedron $(N=20)$. Note that for $N>6$, also other rotor positions exist that yield moments of inertia that are rotationally invariant. For example for $N=8$, any rotor configuration with the rotor positions constrained to the vertices of two arbitrarily aligned tetrahedra with coinciding centers results in a rotationally invariant moment of inertia. If the rotor positions are constrained to the vertices of two arbitrarily aligned tetrahedra, then the rotors are located pairwise at the vertices of a single tetrahedron (see Fig. 3e, the two tetrahedra coincide) and the rotor disks of each pair air aligned perpendicular to each other. Although the simplified set of attainable thrusts and torques has an inradius that is $15.1 \%$ larger than the optimal rotor configuration with the rotors constrained to the vertices of a cube, it is not realizable in practice due to the intersecting rotor disks. Similar results were also found for $N=12$ and $N=20$ and are therefore not depicted.
Due to the assumption that the rotors can generate thrusts of arbitrarily small magnitude, the inner optimization problem of (9) can be solved efficiently by reformulating the attainable thrust and torque set (5) as a polyhedron [25] of the form

$$
\tilde{\mathcal{V}}=\left\{\boldsymbol{y} \in \mathbb{R}^{6} \mid \tilde{\boldsymbol{A}}_{v} \boldsymbol{y} \preceq \tilde{\boldsymbol{b}}_{v}\right\}
$$

where the rows of the matrix $\tilde{\boldsymbol{A}}_{v}$ encode the orientation of polyhedron's faces, the entries of the vector $\tilde{\boldsymbol{b}}_{v}$ determine the offset of the faces from the origin and $\preceq$ denotes componentwise inequality. If the rows of $\tilde{\boldsymbol{A}}_{v}$ are normalized to unit length, then the maximum radius $r$ is given by the smallest component of $\tilde{\boldsymbol{b}}_{v}$. The number of faces ${ }^{3}$ of the polyhedron (10), or equivalently the number of components of $\tilde{\boldsymbol{b}}_{v}$, is proportional to $\left(\begin{array}{c}N \\ 5\end{array}\right)$, which means that the effort to compute (10) grows with $N ! /(N-5)$ !. As a result, the optimization problem for $N=6$ could be solved in under a minute on a regular laptop computer whereas the solution for $N=20$ took several hours to compute.

Note that the rotor disk normals of all rotor configurations are aligned perpendicular to their position vectors in order to maximize the torque output for a given rotor thrust. In addition, the singular values of the thrust and torque map $\tilde{\boldsymbol{B}}$ for all rotor configurations obtained the maximum attainable value of $\sqrt{N / 3}$ (see $\mathrm{A}$ for details), which implies that the rotor configurations are capable of generating the largest possible thrust and torque output.

Although the minimum rotor thrust magnitude is very small in practice, the fact that the rotors cannot generate thrusts of arbitrarily small magnitudes has a significant consequence: Since the real set of feasible rotor thrusts (1) is disconnected, the set of attainable total thrusts and torques for any rotor configuration with only six rotors is also disconnected. This implies that for the hexarotor configuration shown in Fig. 3a, directions exist in which no thrust and torque can be generated, and hence, the hexarotor configuration is unable to hover at arbitrary attitudes. In order for the set of attainable thrusts and torques to be connected, the thrust and torque map $\tilde{\boldsymbol{B}}$ must have a non-trivial null space, which implies that the vehicle must have more than six rotors. Since the optimal rotor configuration with the minimum amount of rotors is not realizable in practice (see Fig. 3e), we decided on the suboptimal rotor configuration shown in Fig. $3 \mathrm{~b}$ with the rotor positions constrained to the vertices of a cube, which is a special case of two arbitrarily aligned tetrahedra that maximizes the distances between the rotors. The optimal rotor configuration for the positions constrained to the vertices of a cube is illustrated in Fig. 3b, where the rotor positions $\boldsymbol{P}$ and rotor disk normals $\mathbf{N}$ are

$$
\begin{aligned}
\boldsymbol{P} & =\frac{1}{\sqrt{3}}\left[\begin{array}{cccccccc}
1 & -1 & 1 & -1 & 1 & -1 & 1 & -1 \\
1 & 1 & -1 & -1 & 1 & 1 & -1 & -1 \\
1 & 1 & 1 & 1 & -1 & -1 & -1 & -1
\end{array}\right], \\
N & =\left[\begin{array}{cccccccc}
-a & b & -b & a & a & -b & b & -a \\
b & a & -a & -b & -b & -a & a & b \\
c & -c & -c & c & c & -c & -c & c
\end{array}\right],
\end{aligned}
$$

\footnotetext{
${ }^{3}$ The faces of the polyhedron (10) in the six dimensional thrust and torque space are spanned by any five column vectors of the thrust and torque map $\boldsymbol{B}$.
} 


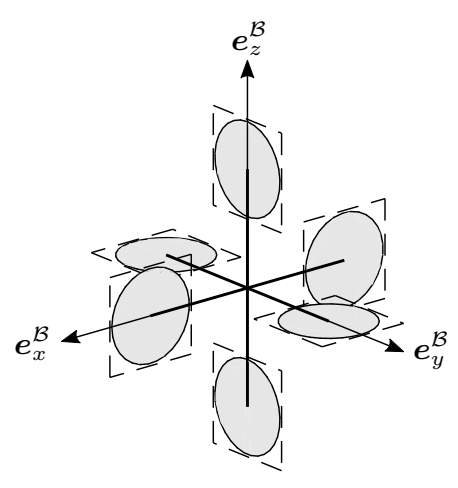

(a) $N=6, r_{\max }=1.41$

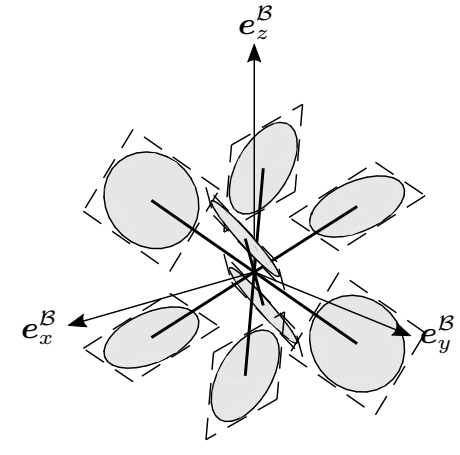

(b) $N=8, r_{\max }=2.31$

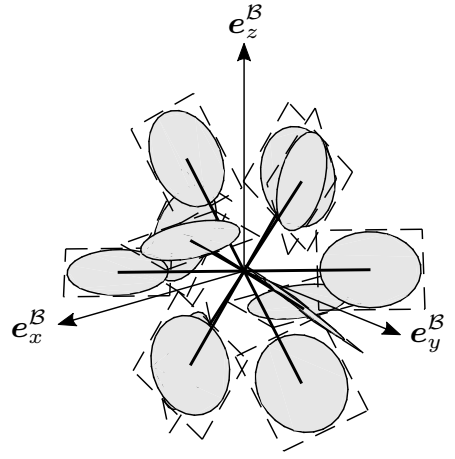

(c) $N=12, r_{\max }=4.24$

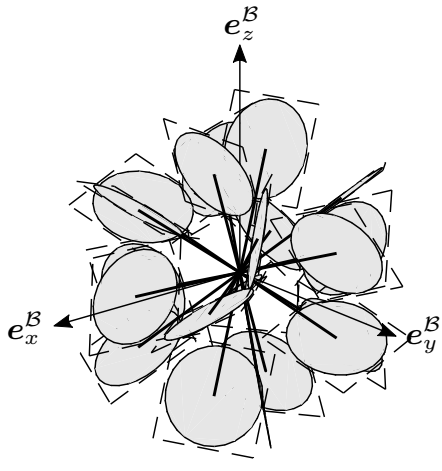

(d) $N=20, r_{\max }=7.88$

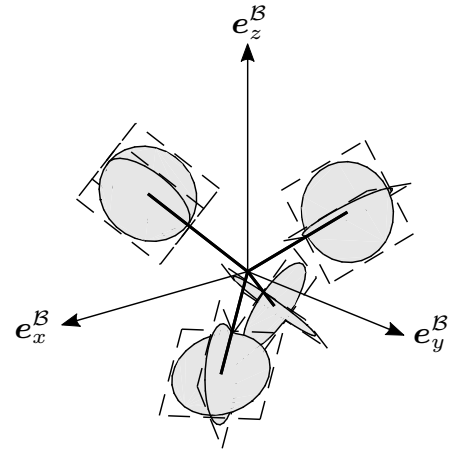

(e) $N=8, r_{\max }=2.66$

Fig. 3: Rotor configurations obtained by numerically solving the optimization problem (9). The configurations (a)-(d) illustrate the optimal solutions with the rotor positions constrained to the vertices of a regular octahedron, a cube, an icosahedron and a dodecahedron. For $N>6$, different rotor configurations exist that yield rotationally invariant moments of inertia. The configuration (e) depicts the optimal rotor configuration with rotor positions constrained to the vertices of two arbitrarily aligned tetrahedra whose centers coincide. Due the intersecting rotor disks, this rotor configuration is not realizable in practice. For a normalized maximum rotor thrust of $f_{\max }=1$, the configurations (a)-(e) are able produce a thrust and torque output in any direction with an Euclidean norm of at least $r_{\max }=1.41,2.31,4.24,7.88$, and 2.66, respectively.

with $a=1 / 2+1 / \sqrt{12}, \quad b=1 / 2-1 / \sqrt{12}$ and $c=1 / \sqrt{3}$. Due to the rotational invariance of the design objective and its constraints, one may expect that all components of the rotor disk normals are given by $\pm 1 / \sqrt{3}$. However, this would yield rotor disk normals that are not aligned perpendicular to their position vectors and hence the rotors would not generate the maximum possible torque for a given rotor thrust. Nevertheless, by expressing the rotor disk normals in a coordinate frame that is rotated by $\pi / 6$ about the vehicle's $z$-axis yields the expected result with the components of the rotor disk normals given by $\pm 1 / \sqrt{3}$.

\section{E. Set of Attainable Thrusts and Torques}

Since the cubic rotor configuration defined by (11) and (12) has eight rotors, its thrust and torque map has a non-trivial null space. However, a non-trivial null space is only a necessary condition in order for the vehicle to be able to generate a total thrust and torque in any direction despite a disconnected set of rotor thrusts, but it does not imply that the vehicle can generate all the thrusts and torques that lie in $\tilde{\mathcal{V}}$.
Let $\mathcal{F}_{\text {rot }}$ denote the set of feasible rotor thrusts,

$$
\mathcal{F}_{\text {rot }}=\left\{\boldsymbol{f}_{\text {rot }} \in \mathbb{R}^{8}\left|f_{\min } \leq\right| f_{\text {rot }, i} \mid \leq f_{\max }\right\} .
$$

The real set of attainable thrusts and torques is then the union of the $2^{8}$ hypercubes defined by (13) projected onto the total thrust and torque space by $\boldsymbol{B}$,

$$
\mathcal{V}=\left\{\boldsymbol{B} \boldsymbol{f}_{\text {rot }} \in \mathbb{R}^{6} \mid \boldsymbol{f}_{\text {rot }} \in \mathcal{F}_{\text {rot }}\right\},
$$

where $\boldsymbol{B}$ denotes the thrust and torque map that includes the effects of aerodynamic rotor drag, i.e.

$$
B=\left[\begin{array}{c}
N \\
P \times N+N K
\end{array}\right],
$$

with $\boldsymbol{K}=\operatorname{diag}\left(\kappa_{1}, \ldots, \kappa_{8}\right)^{4}$. Due to the non-convexity and complicated shape of (14), it is difficult to check if a given

\footnotetext{
${ }^{4}$ The null space of $\tilde{\boldsymbol{B}}$ is spanned by the two null vectors (17) and (18). In order to preserve the structure of the null space when taking the aerodynamic rotor drag into account, the rotors 1-4 need to have the same handedness, and the same holds for the rotors 5-8. The handedness of the rotors is discussed in Section III-B.
} 
total thrust and torque lies within $\mathcal{V}$. In the following, a conservative, convex approximation of the set of attainable thrusts and torques is derived that allows for easy verification of the feasibility of a given thrust and torque.

Claim: If $f_{\max } \geq 7 f_{\min }$, then the cubic octorotor configuration is capable of generating any thrust and torque in the convex set

$$
\mathcal{V}_{\text {conv }}=\left\{\boldsymbol{B} \boldsymbol{f}_{\text {rot }} \in \mathbb{R}^{6}\left|\boldsymbol{f}_{\text {rot }} \in \mathbb{R}^{8},\right| f_{\text {rot }, i} \mid \leq f_{\max }-2 f_{\min }\right\},
$$

i.e. $\mathcal{V}_{\text {conv }} \subseteq \mathcal{V}$.

Proof: The null space of the thrust and torque map $B$ is spanned by the two null vectors

$$
\begin{aligned}
& \boldsymbol{\eta}_{1}=(1,1,1,1,0,0,0,0), \\
& \boldsymbol{\eta}_{2}=(0,0,0,0,1,1,1,1) .
\end{aligned}
$$

It follows directly from the definition of null vectors that biasing the rotor thrusts $1-4$ or $5-8$ by an equal amount does not affect the total thrust and torque, i.e. that all all rotor thrusts

$$
\boldsymbol{f}_{\text {rot }}+\phi_{1} \boldsymbol{\eta}_{1}+\phi_{2} \boldsymbol{\eta}_{2}
$$

generate the same total thrust and torque as $\boldsymbol{f}_{\text {rot }}$ for any biases $\phi_{1}, \phi_{2} \in \mathbb{R}$. Let $\mathcal{F}_{\text {rot,conv }}$ be a convex set of rotor thrusts defined as

$$
\mathcal{F}_{\text {rot,conv }}=\left\{\boldsymbol{f}_{\text {rot }} \in \mathbb{R}^{8}|| f_{\text {rot }, i} \mid \leq f_{\max }-2 f_{\text {min }}\right\} .
$$

In order to proof the claim, it will be shown that for any $f_{\text {rot }} \in \mathcal{F}_{\text {rot,conv }}$ biases $\phi_{1}$ and $\phi_{2}$ exist such that the rotor thrusts are feasible, i.e. $\boldsymbol{f}_{\text {rot }}+\phi_{1} \boldsymbol{\eta}_{1}+\phi_{2} \boldsymbol{\eta}_{2} \in \mathcal{F}_{\text {rot }}$, from which it then follows that $\mathcal{V}_{\text {conv }} \subseteq \mathcal{V}$. Without loss of generality due to symmetry, only the rotors 1-4 are discussed in the following and for the sake of readability, the notation will be misused such that, for example, $f_{\text {rot }} \in \mathcal{F}_{\text {rot }}$ means that the thrusts of rotors 1-4 lie in $\mathcal{F}_{\text {rot }}$. Furthermore, it is assumed that the rotor thrusts 1-4 are sorted in ascending order with rotor 1 generating the smallest thrust and rotor 4 generating the largest thrust.

Consider any rotor thrusts $f_{\text {rot }} \in \mathcal{F}_{\text {rot,conv }}$. It follows from (19) that if the rotor thrusts satisfy

$$
f_{\text {rot }, 4}-f_{\text {rot }, 1} \leq f_{\max }-f_{\text {min }},
$$

then the rotor thrusts can always be biased such that either all rotors generate a positive thrust or a negative thrust and that $\boldsymbol{f}_{\text {rot }}+\phi_{1} \boldsymbol{\eta}_{1} \in \mathcal{F}_{\text {rot }}$. For example, choosing $\phi_{1}=f_{\text {min }}-f_{\text {rot }, 1}$ yields $f_{\text {rot }, 1}+\phi_{1}=f_{\text {min }}$ and $f_{\text {rot }, 4}+\phi_{1} \leq f_{\max }$ (from (21)), and hence the resulting rotor thrusts are feasible (see Fig. 4(a)).

If the constraint (21) is not fulfilled, i.e.

$$
f_{\text {rot }, 4}-f_{\text {rot }, 1}>f_{\max }-f_{\text {min }},
$$

then the rotor thrusts have to be biased such that some rotors generate a positive thrust whereas others generate a negative thrust in order to be feasible. Inserting the assumption that $f_{\max } \geq 7 f_{\min }$ into (22), it follows that $f_{\text {rot }, 4}-f_{\text {rot }, 1} \geq 6 f_{\text {min }}$ and consequently that a pair of subsequent rotor thrusts exist whose difference is larger than $2 f_{\min }$, i.e.

$$
\max \left(f_{\text {rot }, 2}-f_{\text {rot }, 1}, f_{\text {rot }, 3}-f_{\text {rot }, 2}, f_{\text {rot }, 4}-f_{\text {rot }, 3}\right) \geq 2 f_{\min } .
$$

In case of (22), it follows from $f_{\text {rot }} \in \mathcal{F}_{\text {rot,conv }}$ that

$$
\begin{aligned}
& f_{\text {rot }, 1} \in\left[-f_{\max }+2 f_{\min },-f_{\min }\right], \\
& f_{\text {rot }, 4} \in\left[f_{\min }, f_{\max }-2 f_{\min }\right],
\end{aligned}
$$

i.e. only the rotor thrusts $f_{\text {rot }, 2}$ and $f_{\text {rot }, 3}$ can lie in the infeasible range $\left(-f_{\min }, f_{\min }\right)$. Consider the case when $f_{\text {rot }, 2} \in$ $\left(-f_{\min }, f_{\min }\right)$. If

$$
f_{\text {rot }, 2}-f_{\text {rot }, 1} \geq 2 f_{\min },
$$

then the rotor thrusts can be biased by $\phi_{1}=f_{\min }-f_{\text {rot, } 2}$ resulting in the feasible rotor thrusts $\boldsymbol{f}_{\text {rot }}+\phi_{1} \boldsymbol{\eta}_{1} \in \mathcal{F}_{\text {rot }}$ (see Fig. 4(b)), with $\phi_{1} \in\left(0,2 f_{\min }\right)$. In particular, evaluating (19) yields

$$
\begin{aligned}
& f_{\mathrm{rot}, 1}+\phi_{1} \in\left[-f_{\max }+2 f_{\min },-f_{\min }\right], \\
& f_{\mathrm{rot}, 2}+\phi_{1}=f_{\min }, \\
& f_{\mathrm{rot}, 3}+\phi_{1} \in\left[f_{\min }, f_{\max }\right], \\
& f_{\mathrm{rot}, 4}+\phi_{1} \in\left[f_{\min }, f_{\max }\right] .
\end{aligned}
$$

If (26) is not satisfied but instead it holds that

$$
f_{\text {rot }, 3}-f_{\text {rot }, 2} \geq 2 f_{\text {min }},
$$

then the rotor thrusts can be biased by $\phi_{1}=-f_{\min }-f_{\text {rot, } 2}$, with $\phi_{1} \in\left(-2 f_{\min }, 0\right)$, in order to make them feasible (see Fig. 4(c)). In this case, evaluating (19) results in

$$
\begin{aligned}
& f_{\mathrm{rot}, 1}+\phi_{1} \in\left[-f_{\max },-f_{\min }\right], \\
& f_{\mathrm{rot}, 2}+\phi_{1}=-f_{\min }, \\
& f_{\mathrm{rot}, 3}+\phi_{1} \in\left[f_{\min }, f_{\max }-2 f_{\min }\right], \\
& f_{\mathrm{rot}, 4}+\phi_{1} \in\left[f_{\min }, f_{\max }-2 f_{\min }\right] .
\end{aligned}
$$

Finally, if neither (26) nor (31) are satisfied, then, according (23), it must hold that

$$
f_{\text {rot }, 4}-f_{\text {rot }, 3} \geq 2 f_{\min } .
$$

In this case, biasing the rotor thrusts by $\phi_{1}=-f_{\min }-f_{\text {rot }, 3}$, with $\phi_{1} \in\left(-4 f_{\min }, 0\right)$, yields the feasible rotor thrusts

$$
\begin{aligned}
& f_{\mathrm{rot}, 1}+\phi_{1} \in\left[-f_{\max },-f_{\min }\right], \\
& f_{\mathrm{rot}, 2}+\phi_{1} \in\left[-f_{\max },-f_{\min }\right], \\
& f_{\mathrm{rot}, 3}+\phi_{1}=-f_{\min }, \\
& f_{\mathrm{rot}, 4}+\phi_{1} \in\left[f_{\min }, f_{\max }-2 f_{\min }\right],
\end{aligned}
$$

where it was used that $f_{\text {rot }, 3}-f_{\text {rot }, 1}<4 f_{\text {min }}$ (see Fig. 4(d)).

Due to symmetry, the same argumentation can be made if $f_{\text {rot }, 3} \in\left(-f_{\min }, f_{\min }\right)$, and therefore for all rotor thrusts $f_{\text {rot }} \in \mathcal{F}_{\text {rot,conv }}$ a bias $\phi_{1} \in \mathbb{R}$ can be found such that $\boldsymbol{f}_{\text {rot }}+\phi_{1} \boldsymbol{\eta}_{1} \in \mathcal{F}_{\text {rot }}$ without affecting the total thrust and torque generated by the rotors, and hence $\mathcal{V}_{\text {conv }} \subseteq \mathcal{V}$.

It follows from the proof above that $\mathcal{V}_{\text {conv }}$ is not only a subset of $\mathcal{V}$, but that both sets also share the same insphere. Furthermore, it is straightforward to see that $\mathcal{V}$ is a subset of

$$
\left\{\boldsymbol{B} \boldsymbol{f}_{\text {rot }} \in \mathbb{R}^{6}\left|\boldsymbol{f}_{\text {rot }} \in \mathbb{R}^{8},\right| f_{\text {rot }, i} \mid \leq f_{\max }\right\}
$$

and that both sets share the same circumsphere. The loss in the maximum attainable thrust and torque due of the convex approximation of $\mathcal{V}_{\text {conv }}$ can therefore be bounded by 
(a)

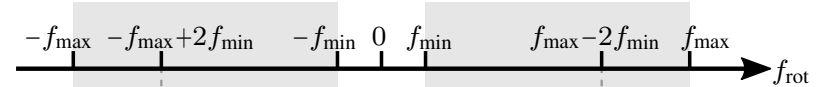

(b)

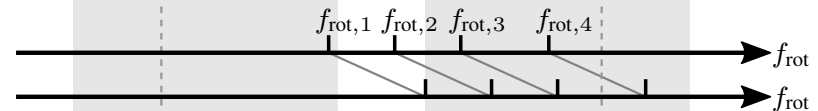

(c)

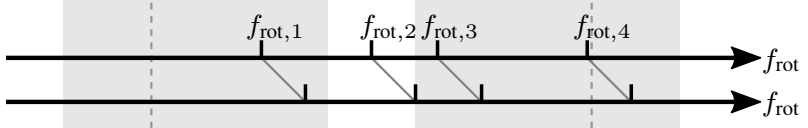

(d)
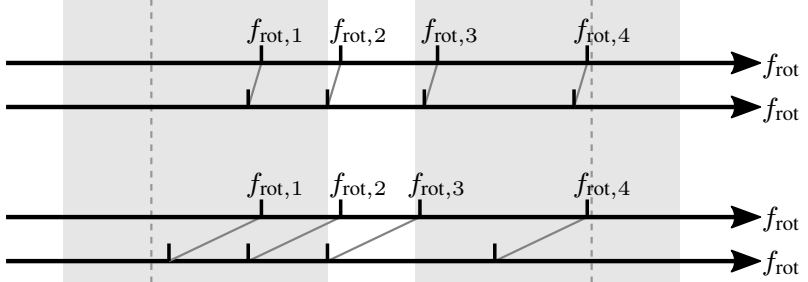

TABLE I: Physical attributes of the multirotor vehicle.

\begin{tabular}{cll}
\hline Symbol & Description & Value \\
\hline $\mathrm{m}$ & Total mass & $0.892 \mathrm{~kg}$ \\
& motors and rotors & $0.448 \mathrm{~kg}$ \\
& battery & $0.205 \mathrm{~kg}$ \\
& chassis & $0.170 \mathrm{~kg}$ \\
& electronics & $0.046 \mathrm{~kg}$ \\
& others (screws, cables, ...) & $0.023 \mathrm{~kg}$ \\
$J_{x x}$ & Vehicle's moment of inertia about $\boldsymbol{e}_{x}^{\mathcal{B}}$ & $1.16 \times 10^{-2} \mathrm{~kg} \mathrm{~m}^{2}$ \\
$J_{y y}$ & Vehicle's moment of inertia about $\boldsymbol{e}_{y}^{\mathcal{B}}$ & $1.13 \times 10^{-2} \mathrm{~kg} \mathrm{~m}^{2}$ \\
$J_{z z}$ & Vehicle's moment of inertia about $\boldsymbol{e}_{z}^{\mathcal{B}}$ & $1.13 \times 10^{-2} \mathrm{~kg} \mathrm{~m}^{2}$ \\
$J_{\text {rot }}$ & Rotor's moment of inertia about $\boldsymbol{n}_{i}$ & $3.41 \times 10^{-5} \mathrm{~kg} \mathrm{~m}^{2}$ \\
$\left\|\boldsymbol{p}_{i}\right\|$ & Distance of $i$-th rotor from center & $0.184 \mathrm{~m}$ \\
$f_{\min }$ & Minimum (absolute) rotor thrust & $0.15 \mathrm{~N}$ \\
$f_{\max }$ & Maximum (absolute) rotor thrust & $6.25 \mathrm{~N}$ \\
\hline
\end{tabular}

The vehicle's moment of inertia $\boldsymbol{J}=\operatorname{diag}\left(J_{x x}, J_{y y}, J_{z z}\right)$ includes the moment of inertia of the rotors and was obtained from a detailed CAD model.

Fig. 4: Any set of rotor thrusts that satisfies $\left|f_{\text {rot }, i}\right| \leq f_{\max }-2 f_{\min }, \forall i \in\{1, \ldots, 4\}$, can be made feasible without affecting the total thrust and torque by biasing each rotor with an equal amount of thrust. The gray shaded area represents the set of feasible rotor thrusts and the dashed lines indicate the boundaries $\pm\left(f_{\max }-2 f_{\min }\right)$.

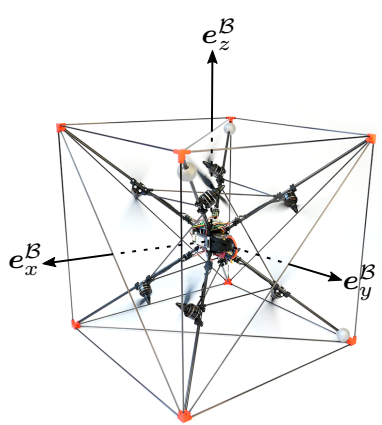

Fig. 5: The omni-directional multirotor vehicle with the rotor configuration designed in Section II. The rotor configuration is embedded in a cubic chassis that protects the rotors from collisions and provides structural rigidity.

$2 f_{\min } / f_{\max }$. Due to the large ratio of $f_{\max }$ to $f_{\min }$ for the propulsion systems typically used in multirotor vehicles, the loss is usually very small, and hence the set $\mathcal{V}_{\text {conv }}$ will be referred to as the set of attainable total thrusts and torques in the remainder of this paper and its subscript will be dropped. The set of attainable thrusts (at zero torque) and the set of attainable torques (at thrusts sufficiently large to hover at any attitude) of a prototype vehicle with the cubic rotor configuration are depicted in Fig. 6a and Fig. 6b, respectively.

\section{SYSTEM DESCRIPTION}

This section describes the implementation of an omnidirectional multirotor vehicle with the cubic rotor configuration (11) and (12). An image of the vehicle is shown in Fig 5. The octorotor configuration is enclosed by a cubic chassis with a characteristic edge length of $0.45 \mathrm{~m}$. The vehicle has a total mass of $0.892 \mathrm{~kg}$ and its rotors enable it to generate a total thrust of at least $19.4 \mathrm{~N}$ in any direction.

In the following, the vehicle's chassis, actuation and electronics are described in more detail.

\section{A. Chassis}

The main purpose of the chassis is to hold all components in place and protect the rotors from collisions. It must be lightweight to enable agile flight and must interfere as little as possible with the rotors' air streams. Furthermore, it must be rigid enough to accommodate for the large internal forces caused by the non-planar rotor configuration without deforming and yet durable enough to withstand crashes. To meet these requirements, a cubic chassis is constructed comprising composite carbon tubes, for their light weight and stiffness, and additively manufactured nylon (PA2200) parts, which exhibit few fabrication constraints. The chassis is composed of three main components: a centerpiece, which houses the battery and holds all electronics, an outer cubic frame, which protects the internal components and provides structural rigidity, and eight composite carbon tubes that connect the outer frame with the centerpiece and hold the rotor mounts.

Due to the chassis' cubic shape, the vehicle's center of mass is located at the chassis' geometrical center and the vehicle's moment of inertia remains almost rotationally invariant. The rotor mounts are placed at a distance of $0.184 \mathrm{~m}$ from the center and are connected together by thin composite carbon tubes, which serve the purpose of increasing the rigidity, but more importantly constrain the rotor positions and orientations to the configuration defined by (11) and (12). The total weight of the chassis is $0.170 \mathrm{~kg}$, which corresponds to $19.1 \%$ of the

Table I lists the vehicle's most important physical attributes.

\section{B. Actuation}

The vehicle's eight rotors are driven by off-the-shelf 14-pole outrunner brushless direct-current (DC) motors (MRM Titan total vehicle weight. 


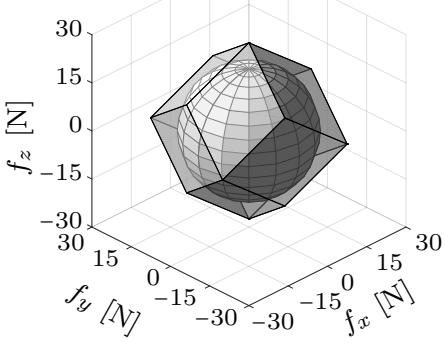

(a)

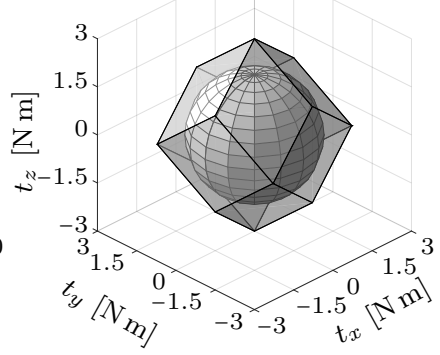

(b)
Fig. 6: The left figure (a) shows the set of attainable thrusts for zero torque, which is described by a rhombic dodecahedron with an inradius of $19.4 \mathrm{~N}$. The right figure (b) shows the set of attainable torques when generating a thrust of magnitude $m g$ in any direction, where $m$ denotes the vehicle's mass and $g$ denotes the gravitational constant, i.e. $g=9.81 \mathrm{~m} / \mathrm{s}^{2}$. The set of attainable torques is described by a similar dodecahedron as (a) and has an inradius of $1.9 \mathrm{~N} \mathrm{~m}$, however, it is slightly skewed due to the contribution of the aerodynamic drag of the rotors to the total torque. When generating zero thrust, the set of attainable torques does not change its shape, but becomes larger and has an inradius of $3.4 \mathrm{~N} \mathrm{~m}$.

2208-1100KV), which are controlled by eight ESCs (DYS SN20A) running the SimonK open-source firmware [26]. A $1800 \mathrm{~mA} \mathrm{~h}$ four-cell lithium-ion polymer battery (Thunder Power RC TP1800-4SM70), which is located in the center of the vehicle, powers the brushless DC motors. The battery is capable of continuously providing power up to $1864 \mathrm{~W}$, which suffices to run all motors at their maximum angular velocity, and the battery's capacity lasts for approximately seven minutes of hover flight. The motors are directly connected to a symmetric two-blade fixed-pitch rotor (Graupner 3D $8 \times 4.5$ ), which generates a minimum thrust of $0.15 \mathrm{~N}$ and a maximum thrust of $6.25 \mathrm{~N}$ at the motor's minimum and maximum angular velocity, respectively. In order to obtain the nicely structured null space (17) and (18) of the thrust and torque map $B$, the rotors 1-4 need to have the same handedness and the rotors 5-8 need to have the same handedness. Since the rotor disk normals of opposite rotors have the same rotor disk normal, i.e. rotors for which $\boldsymbol{p}_{i}=-\boldsymbol{p}_{j}, i \neq j$, holds, it was decided that opposite rotors should have different handedness such that the sum of their angular momentum is typically zero $^{5}$. Motors 1-4 thus drive rotors that generate positive thrusts when the motors are rotating clockwise, and motors 58 drive rotors that generate positive thrust when the motors are rotating counter-clockwise. The set of attainable thrusts when generating zero torque and the set of attainable torques when generating thrusts sufficiently large to hover at any attitude are shown in Fig. 6a and Fig. 6b, respectively.

\footnotetext{
${ }^{5}$ This assumes that the vehicle mainly needs to generate a thrust and only very little torque, such as, for example, during hovering. In this case, it is easy to see that the control allocation strategy of Section V-E commands opposite rotors to generate an equal amount of thrust in the long term and hence opposite rotors rotate at an equal angular velocity.
}

The ESCs control the commutation steps of the motors, and allow setting the voltage that is applied to each individual motor by pulse-width modulating the battery voltage. The duty-cycle of the pulse-width modulation can be set at a rate of up to $4 \mathrm{kHz}$ using the oneshot 125 protocol. By reversing the sequence of the commutation steps, the ESC can drive the motor in either a clockwise or a counter-clockwise direction.

It is important to note that the ESCs do not directly control the motors' angular velocities, but only the applied voltages. In order to control the motors' angular velocities in a closed loop and thereby achieve more accurate control and faster response times, a digital-type tachometer has been installed that provides angular velocity feedback. In particular, the ESC's firmware has been modified such that a pin of the ESC's microcontroller is toggled between high and low every third commutation step, i.e. twice per electrical revolution. The time between two subsequent toggles is measured by the vehicle's flight computer in order to calculate the motor's angular velocity. Note that this only allows control of the motor's angular velocity within a range where the rate of toggling is high enough to provide sufficient bandwidth. However, the minimal angular rate of the motor for which the zero-crossings of the back electromotive force could reliably be detected was found to be $178 \mathrm{rad} / \mathrm{s}$. The motor used has 14 poles and therefore 7 electrical revolutions are required for one mechanical revolution. Feedback of the angular velocity is thus obtained at a minimum rate of $396.7 \mathrm{~Hz}$, which was found to be sufficiently fast for the desired bandwidth.

\section{Electronics}

The primary electrical component is a flight computer that is custom-designed to meet the vehicle's on-board sensing, communication and computation requirements. A $168 \mathrm{MHz}$ ARM-based 32-bit microcontroller (ST Microelectronics ARM Cortex-M4 STM32F405x) on the flight computer board handles all the required on-board computation and runs an in-house developed firmware based on the real-time operating system NuttX [27]. The flight computer is rigidly mounted to the chassis' center piece and is equipped with a nine-axis MEMS inertial measurement unit (InvenSense MPU-9250) that is capable of sampling the vehicle's angular velocity and translational acceleration at a rate of $1 \mathrm{kHz}$ with a resolution of $1.06 \times 10^{-3} \mathrm{rad} / \mathrm{s}$ and $4.79 \times 10^{-3} \mathrm{~m} / \mathrm{s}^{2}$, respectively. The inertial measurement unit (IMU) communicates its information to the microcontroller over a serial peripheral interface (SPI). A power board handles the power distribution from the battery to the flight computer and ESCs. A 12-bit analog to digital converter (ADC), which is available on the microcontroller, is used to measure the battery voltage. The measured battery voltage is used to accurately determine the duty-cycle of the pulse-width modulated battery voltage that is applied to the brushless DC motors by the ESCs. The flight computer is able to receive commands from a base station through a low-latency radio module (Laird RM024), and can transmit non-critical telemetry data to a base station over a regular $2.4 \mathrm{GHz}$ wireless module (Microchip RN131). Both communication modules are connected to the microcontroller through a standard universal asynchronous receiver/transmitter (UART) interface. 


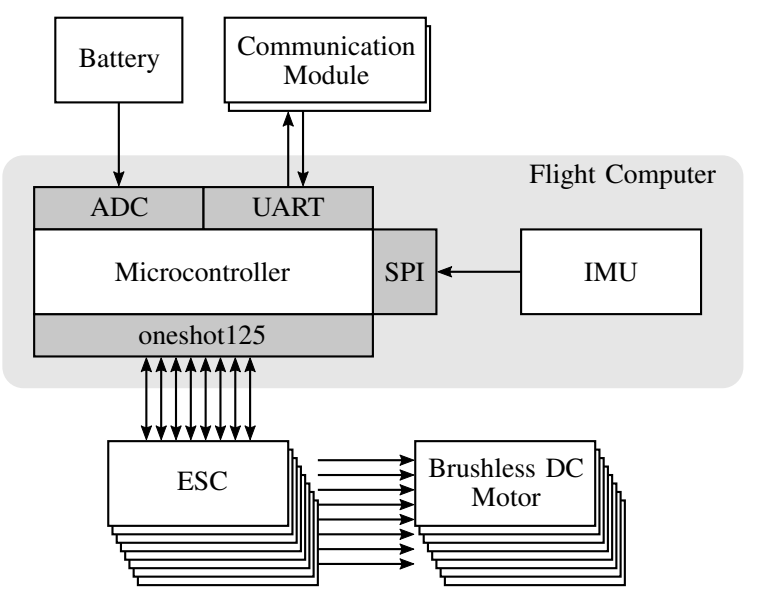

Fig. 7: Overview of the vehicle's principal electrical components. A custom-designed flight computer that is equipped with a 32-bit microcontroller and a nine-axis IMU handles all the on-board computation required to execute the flight commands received. The microcontroller measures the motors' angular velocities and the battery voltage in order to compute the duty-cycle commands that are sent to the ESCs. Two communication modules are used to receive commands from a base station and transmit telemetry data.

An overview of the vehicle's principal electrical components is given in Fig. 7.

\section{System ModelLing}

This section presents models for the thrust and torque generated by the rotors, the motor dynamics and the vehicle's position and attitude dynamics. The motor dynamics are modelled to be independent of the vehicle's position and attitude dynamics because the motor dynamics are several orders of magnitude faster than the position and attitude dynamics and are controlled by high-bandwidth controllers. The impact of the vehicle's position and attitude dynamics on the motor dynamics are therefore treated as disturbances that are assumed to be taken care of by the motor controllers.

\section{A. Rotor Thrust and Torque}

The omni-directional multirotor vehicle can generate thrust and torque by spinning its rotors. The thrust generated by the $i$-th rotor can be modelled using momentum-blade element theory [28] and results in an expression proportional to the square of the rotor's angular velocity $\Omega_{i}$,

$$
f_{\text {rot }, i}=c_{f} \operatorname{sgn}\left(\Omega_{i}\right) \Omega_{i}^{2},
$$

where $c_{f}$ denotes the rotor specific thrust coefficient and $\operatorname{sgn}\left(\Omega_{i}\right)$ is defined as

$$
\operatorname{sgn}\left(\Omega_{i}\right)= \begin{cases}1, & \text { if } \Omega_{i} \geq 0 \\ -1, & \text { if } \Omega_{i}<0\end{cases}
$$

Every spinning rotor also causes a torque due to the aerodynamic drag of its rotor blades that can be modelled similarly to (42),

$$
t_{\mathrm{rot}, i}=-c_{t} \operatorname{sgn}\left(\Omega_{i}\right) \Omega_{i}^{2},
$$

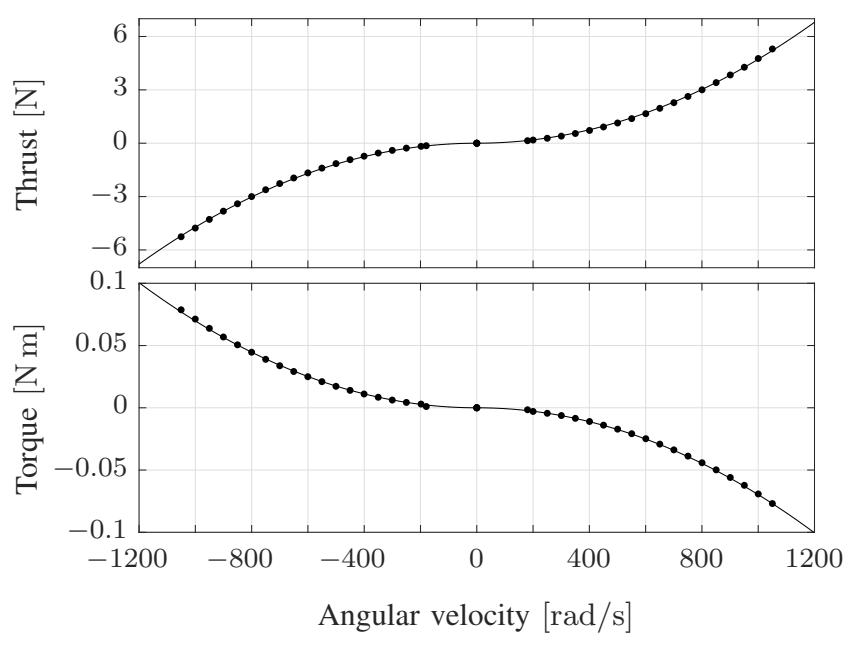

Fig. 8: The thrust and torque coefficients $c_{f}$ and $c_{t}$ are experimentally determined using load cell measurements. Each dot denotes the average of 500 samples taken at a given angular velocity and the solid lines denote curve fits of the form (42) and (44), respectively. The root-mean-square error (RMSE) of the thrust and torque fit for this experiment are $3.2 \times 10^{-2} \mathrm{~N}$ and $5.2 \times 10^{-4} \mathrm{~N} \mathrm{~m}$, respectively.

TABLE II: Rotor parameters.

\begin{tabular}{cll}
\hline Symbol & Description & Value \\
\hline$c_{f}$ & Thrust coefficient & $4.721 \times 10^{-6} \mathrm{~N} /(\mathrm{rad} / \mathrm{s})^{2}$ \\
$c_{t}$ & Torque coefficient & $6.976 \times 10^{-8} \mathrm{Nm} /(\mathrm{rad} / \mathrm{s})^{2}$ \\
$\kappa$ & Thrust-to-drag ratio & $1.478 \times 10^{-2} \mathrm{Nm} / \mathrm{N}$ \\
\hline
\end{tabular}

where $c_{t}$ denotes the rotor specific torque coefficient.

The rotor's thrust and torque coefficients $c_{f}$ and $c_{t}$ are functions of the rotor's geometry, air density and incoming air speed. While the former two can be assumed to be constant, it is apparent from the rotor configuration that the incoming air speed varies considerably depending on the thrust generated by the other rotors and to a smaller extent on the vehicle's velocity. However, for the sake of simplicity, it is assumed that the air surrounding the rotors is stationary such that $c_{f}$ and $c_{t}$ can be modelled as constants, resulting in the constant thrust-to-drag ratio $\left|\kappa_{i}\right|=c_{t} / c_{f}$ that was used in (3).

The rotor's thrust and torque coefficients were identified experimentally for the static case using a load cell. The measured rotor thrust and torque are plotted against the rotor's angular velocity in Fig. 8, and the rotor's characteristic attributes are listed in Table II.

\section{B. Motor Dynamics}

Each rotor is driven by a brushless DC motor with permanent magnets rotating around a fixed armature with three phases. An ESC is used to control the motor's commutation, such that at any time two phases are conducting while the third phase is floating. This effectively hides many of the complexities of a brushless DC motor, allowing it to be 
modelled as a conventional DC motor with its dynamics described by the simplified electrical equation [29]

$$
u_{i}=R i+L \frac{d i}{d t}+k_{e} \Omega_{i},
$$

where $u_{i}$ is the voltage applied to phases of the $i$-th motor, $i$ is the current running through the phases, $R$ is the phase-tophase resistance, $L$ is the phase-to-phase inductance, and $k_{e} \Omega_{i}$ is the internally generated voltage that is proportional to the rotor's angular velocity $\Omega_{i}$ and scaled with the motor specific voltage constant $k_{e}$. For the sake of readability, the subscript $i$ is omitted when referring to the current running through the $i$-th motor. The motor's mechanical dynamics are governed by

$$
J_{\text {rot }} \dot{\Omega}_{i}=k_{e} i-t_{\text {load }}\left(\Omega_{i}\right),
$$

where $J_{\text {rot }}$ is the moment of inertia of the rotor and the rotating part of the motor, $k_{e} i$ is the torque generated by the motor, which is proportional to the current $i$ and scaled by the positive motor constant $k_{e}$, and $t_{\text {load }}\left(\Omega_{i}\right)$ is the motor load torque due to friction, viscous damping and the aerodynamic drag of the rotor (44). The brushless DC motors used in multirotor vehicles are typically designed to have a very low phase-tophase inductance, which implies that the motor's electrical dynamics are much faster than its mechanical dynamics and can be neglected. The motor dynamics can therefore be simplified by inserting (45) into (46) and setting the time derivative of the current to zero, resulting in the nonlinear first-order differential equation

$$
\dot{\Omega}_{i}=-\frac{1}{J_{\text {rot }}}\left(t_{\text {load }}\left(\Omega_{i}\right)+\frac{k_{e}^{2}}{R} \Omega_{i}\right)+\frac{k_{e}}{J_{\text {rot }} R} u_{i} .
$$

Instead of modelling the motor load torque from first principles and identifying all motor parameters, the motor dynamics are characterized experimentally. The voltage $\bar{u}$ required for the motor to achieve a steady-state angular velocity of $\Omega_{i}$ is depicted in Fig. 9 and reads as

$$
\bar{u}\left(\Omega_{i}\right)=\frac{R}{k_{e}} t_{\text {load }}\left(\Omega_{i}\right)+k_{e} \Omega_{i} .
$$

The steady-state voltage can be approximated by a quadratic polynomial,

$$
\bar{u}\left(\Omega_{i}\right) \approx c_{u, 2} \Omega_{i}^{2}+c_{u, 1} \Omega_{i}+c_{u, 0},
$$

which is expected due to the quadratic dependency of the rotor drag on the angular velocity, the linear term in (48) and the viscous and static friction of the rotor. The motor's frequency response from applied voltage to angular velocity at different operating points is shown in Fig. 10. A sinusoidally varying voltage was applied to the motor with different voltage offsets ranging from $3 \mathrm{~V}$ to $11 \mathrm{~V}$, and the resulting angular velocity amplitude around the steady-state angular velocity was measured at $1 \mathrm{kHz}$. The angular velocity response is characterized by a first-order system for frequencies up to $20 \mathrm{rad} / \mathrm{s}$, which confirms that the electrical dynamics can be neglected. The scalar $k_{e} /\left(J_{\text {rot }} R\right)$ can be identified from the frequency response's steady-state gain and time constant. Table III lists all relevant motor parameters.

Note that reversing the direction of rotation of the motor is achieved by reversing the order of the commutation sequence

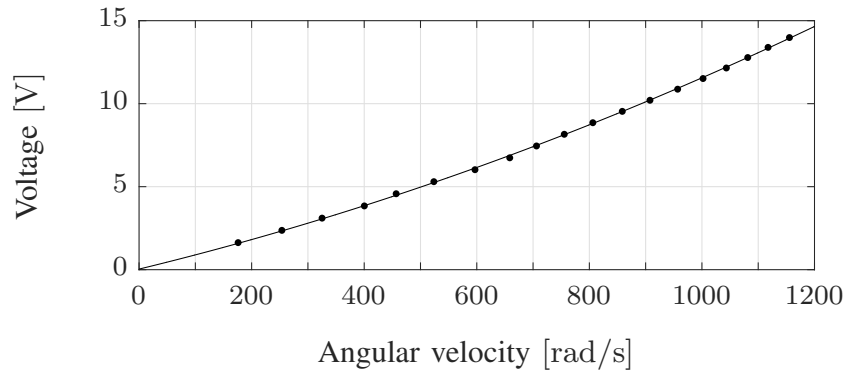

Fig. 9: The voltage required for the motor to achieve a steadystate angular velocity was experimentally identified. Each dot represents the average of 500 angular velocity measurements for a given voltage. The solid line represents a quadratic polynomial fit with a RMSE of $6.00 \times 10^{-2} \mathrm{~V}$.

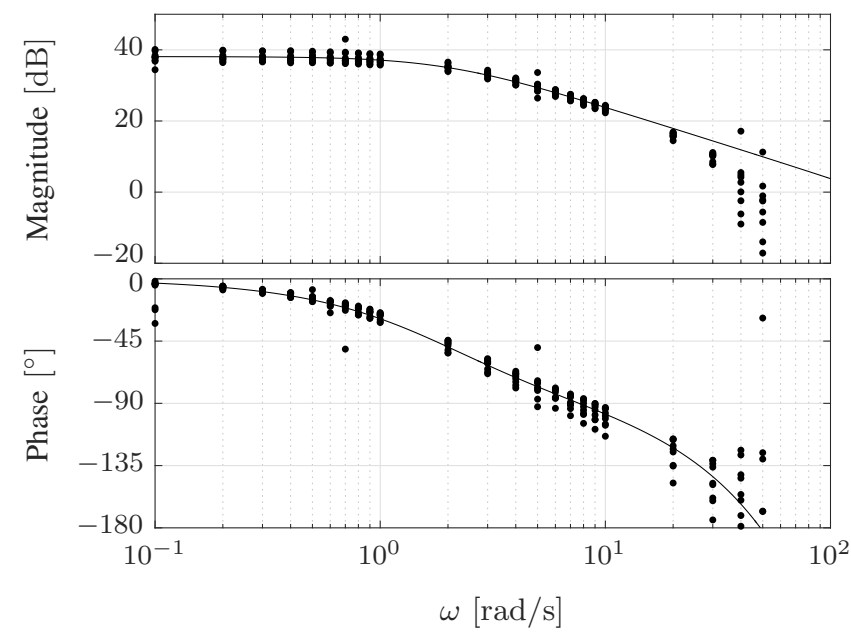

Fig. 10: The frequency response of the motor from voltage input to angular velocity approximates a first-order transfer function with a gain of $80.3(\mathrm{rad} / \mathrm{s}) / \mathrm{V}$, a time constant of $8.1 \times 10^{-2} \mathrm{~s}$ and a delay of $4.7 \times 10^{-3} \mathrm{~s}$.

TABLE III: Motor parameters.

\begin{tabular}{lll}
\hline Symbol & Description & Value \\
\hline$c_{u, 2}$ & Feed-forward voltage coefficient & $3.292 \times 10^{-6} \mathrm{~V} /(\mathrm{rad} / \mathrm{s})^{2}$ \\
$c_{u, 1}$ & Feed-forward voltage coefficient & $8.230 \times 10^{-3} \mathrm{~V} /(\mathrm{rad} / \mathrm{s})$ \\
$c_{u, 0}$ & Feed-forward voltage coefficient & $3.580 \times 10^{-2} \mathrm{~V}$ \\
$\frac{k_{e}}{J_{\mathrm{rot}} R}$ & Motor voltage gain & $9.926 \times 10^{2}\left(\mathrm{rad} / \mathrm{s}^{2}\right) / \mathrm{V}$ \\
\hline
\end{tabular}

and not by applying a negative voltage $u_{i}$. While the direction of rotation is being reversed, the above model does not describe the motor dynamics well. In this case, the ESC executes the commutation sequence in open-loop until the back electromotive force can be measured reliably, and as a consequence, the simplified electrical equation (45) does not hold. Since the motor dynamics are substantially slower when the commutations are executed in open-loop, a control allocation strategy to avoid reversing the direction of rotation is devised in Section V-E. 


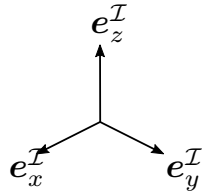

Fig. 11: Illustration of the omni-directional multirotor vehicle with its body-fixed coordinate frame $\mathcal{B}$. The vehicle can generate a total thrust $\boldsymbol{f}$ and a torque $\boldsymbol{t}$ in any desired direction by adjusting the thrust of its eight rotors. In addition, gravity $\boldsymbol{g}$ is acting upon the vehicle.

\section{Vehicle Position and Attitude Dynamics}

The omni-directional multirotor vehicle is modelled as a rigid body with mass $m$ and moment of inertia $\boldsymbol{J}=\operatorname{diag}\left(J_{x x}, J_{y y}, J_{z z}\right)$, where $\boldsymbol{J}$ includes the moments of inertia of the rotors, which were modelled as disks. A list of the vehicle's physical parameters is given in Table I.

The vehicle's translational degrees-of-freedom are described by the position of its center of mass $\boldsymbol{p}=\left(p_{x}, p_{y}, p_{z}\right)$ with respect to an inertial frame $\mathcal{I}$ as illustrated in Fig. 11. The rotational degrees-of-freedom are parametrized using a unit quaternion $\boldsymbol{q}=\left(q_{0}, \tilde{\boldsymbol{q}}\right)$ that describes the rotation between the inertial frame $\mathcal{I}$ and the body-fixed coordinate frame $\mathcal{B}$, where $q_{0}$ is the quaternion's scalar component and $\tilde{\boldsymbol{q}}=\left(q_{1}, q_{2}, q_{3}\right)$ its vector component [30]. The evolution of the attitude in time is determined by the vehicle's angular velocity $\omega$, which is expressed in the body-fixed coordinate frame $\mathcal{B}$, and reads as

$$
\dot{\boldsymbol{q}}=\frac{1}{2}\left[\begin{array}{c}
0 \\
\boldsymbol{\omega}
\end{array}\right] \otimes \boldsymbol{q},
$$

where $\otimes$ denotes the quaternion multiplication operator,

$$
\boldsymbol{q} \otimes \boldsymbol{r}=\left[\begin{array}{cc}
q_{0} & -\tilde{\boldsymbol{q}}^{T} \\
\tilde{\boldsymbol{q}} & q_{0} \boldsymbol{I}_{3 \times 3}-[\tilde{\boldsymbol{q}}]_{\times}
\end{array}\right] \boldsymbol{r},
$$

$\boldsymbol{I}_{3 \times 3}$ is the identity matrix and $[\tilde{\boldsymbol{q}}]_{\times}$is the skew-symmetric cross product matrix representation of $\tilde{\boldsymbol{q}}$,

$$
[\tilde{\boldsymbol{q}}]_{\times}=\left[\begin{array}{ccc}
0 & -q_{3} & q_{2} \\
q_{3} & 0 & -q_{1} \\
-q_{2} & q_{1} & 0
\end{array}\right] .
$$

It is assumed that the multirotor vehicle operates mostly at small velocities such that the aerodynamic drag on its fuselage is negligible. The vehicle's position and attitude dynamics are then given by the Newton-Euler equations [31],

$$
\begin{gathered}
m \ddot{\boldsymbol{p}}=\boldsymbol{R}(\boldsymbol{q})^{-1} \boldsymbol{f}-m \boldsymbol{g} \\
\boldsymbol{J} \dot{\boldsymbol{\omega}}+\sum_{i=1}^{8} J_{\mathrm{rot}} \dot{\Omega}_{i} \boldsymbol{n}_{i}=\boldsymbol{t}-\boldsymbol{\omega} \times\left(\boldsymbol{J} \boldsymbol{\omega}+\sum_{i=1}^{8} J_{\mathrm{rot}} \Omega_{i} \boldsymbol{n}_{i}\right),
\end{gathered}
$$

where $\boldsymbol{R}(\boldsymbol{q})^{-1}$ is the rotation matrix that maps a vector from the body-fixed coordinate frame $\mathcal{B}$ to the inertial frame $\mathcal{I}$,

$$
\boldsymbol{R}(\boldsymbol{q})=\left(q_{0}^{2}-\tilde{\boldsymbol{q}}^{T} \tilde{\boldsymbol{q}}\right) \boldsymbol{I}_{3 \times 3}+2\left(\tilde{\boldsymbol{q}} \tilde{\boldsymbol{q}}^{T}-q_{0}[\tilde{\boldsymbol{q}}]_{\times}\right),
$$

$\boldsymbol{g}=(0,0,9.81) \mathrm{m} / \mathrm{s}^{2}$ denotes the acceleration due to gravity, and $\boldsymbol{f}$ and $\boldsymbol{t}$ are the vehicle's total thrust and torque defined by (2) and (3), respectively. Because the rotors' moment of inertia $J_{\text {rot }}$ is several orders of magnitude smaller than the vehicle's moment of inertia $\boldsymbol{J}$ (see Table I), it is furthermore assumed that the term $J_{\text {rot }} \dot{\Omega}_{i} \boldsymbol{n}_{i}$ in (54) is negligible. Note that due to the large rotor angular velocities $\Omega_{i}$, the angular momentum of the rotors might still be comparable to that of the vehicle, such that the term $J_{\text {rot }} \Omega_{i} \boldsymbol{n}_{i}$ may not be negligible compared to $\boldsymbol{J} \boldsymbol{\omega}$.

\section{Control Design}

This section presents a control strategy that allows the omnidirectional multirotor vehicle to simultaneously track a desired position and attitude trajectory $\boldsymbol{p}_{\mathrm{des}}(t)$ and $\boldsymbol{q}_{\mathrm{des}}(t)$, which are generated for example using [32].

The control strategy is based on a cascaded control scheme, and hence the control design is split into the design of several controllers of lower-order dynamic systems. The controllers of the lower-order systems are designed under the assumption that the underlying control loops can track set point changes perfectly, i.e. without any dynamics or delay.

\section{A. Control System Overview}

An overview of the full cascaded control system is depicted in Fig. 12. Because the vehicle's position and attitude dynamics are decoupled, the task of tracking position and attitude trajectories is performed by two separate control loops. First, thrust and angular velocity commands are computed by a position and an attitude controller, respectively. The commanded angular velocity is subsequently tracked by an inner angular velocity control loop which outputs a torque command. Using the thrust and torque map (15), the individual rotor thrusts required to produce the commanded total thrust and torque, or equivalently the rotor angular velocities, are computed. Finally, the commanded rotor angular velocities are tracked by continuously adjusting the voltage that is applied to the motors.

Note that the angular velocity controller, control allocation and motor controllers run on board, whereas the position and attitude controller run off board. This is due to the experimental setup where an external motion capture system is used to measure the pose of the vehicle (see Section VI-A). The on-board controllers perform feedback control on all the states that are observable from local measurements, thus avoiding sending accurately timed pose information to the vehicle at high rates.

\section{B. Position Control}

The position tracking controller is shaped to respond to position commands in the fashion of a linear, time invariant 


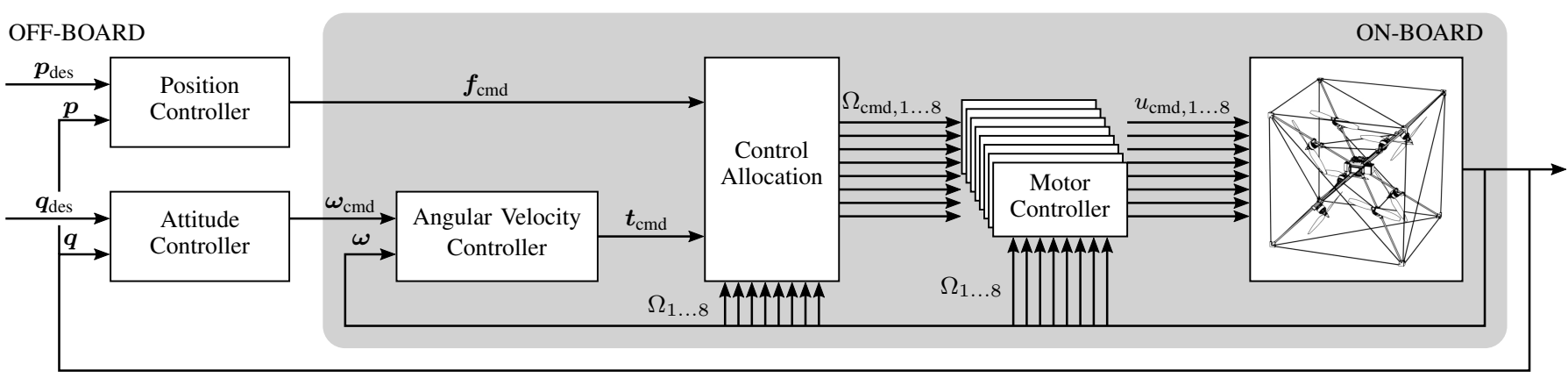

Fig. 12: Overview of the cascaded control system.

second-order system with time constant $\tau_{\text {pos }}$ and damping ratio $\zeta_{\text {pos }}$,

$$
\ddot{\boldsymbol{p}}=\frac{1}{\tau_{\mathrm{pos}}^{2}} \boldsymbol{p}_{\mathrm{err}}+\frac{2 \zeta_{\mathrm{pos}}}{\tau_{\mathrm{pos}}} \dot{\boldsymbol{p}}_{\mathrm{err}}+\ddot{\boldsymbol{p}}_{\mathrm{des}},
$$

where $\boldsymbol{p}_{\text {err }}=\boldsymbol{p}_{\text {des }}-\boldsymbol{p}$ denotes the position error. Using the position dynamics (53), the total thrust required to achieve the desired second-order system response is found to be

$$
\begin{aligned}
\boldsymbol{f}_{\mathrm{cmd}}=m \boldsymbol{R}(\boldsymbol{q})\left(k_{\mathrm{pos}, \mathrm{p}} \boldsymbol{p}_{\mathrm{err}}\right. & +k_{\mathrm{pos}, \mathrm{i}} \int \boldsymbol{p}_{\mathrm{err}} d t \\
& \left.+k_{\mathrm{pos}, \mathrm{d}} \dot{\boldsymbol{p}}_{\mathrm{err}}+\ddot{\boldsymbol{p}}_{\mathrm{des}}+\boldsymbol{g}\right),
\end{aligned}
$$

where an integral term was added to reduce the steady-state effects of disturbances and to compensate for modelling errors. For an integral term with time constant $\tau_{\text {pos }, \mathrm{i}}$, the proportional, integral and derivative gains $k_{\mathrm{pos}, \mathrm{p}}, k_{\mathrm{pos}, \mathrm{i}}$ and $k_{\mathrm{pos}, \mathrm{d}}$ are given by [33]

$$
\begin{aligned}
& k_{\mathrm{pos}, \mathrm{p}}=\frac{1}{\tau_{\mathrm{pos}}^{2}}+\frac{2 \zeta_{\mathrm{pos}}}{\tau_{\mathrm{pos}} \tau_{\mathrm{pos}, \mathrm{i}}}, \\
& k_{\mathrm{pos}, \mathrm{i}}=\frac{1}{\tau_{\mathrm{pos}}^{2} \tau_{\mathrm{pos}, \mathrm{i}}}, \\
& k_{\mathrm{pos}, \mathrm{d}}=\frac{2 \zeta_{\mathrm{pos}}}{\tau_{\mathrm{pos}}}+\frac{1}{\tau_{\mathrm{pos}, \mathrm{i}}} .
\end{aligned}
$$

\section{Attitude Control}

A global asymptotically stable attitude control law similar to [34] is applied that allows for tracking of any desired attitude trajectory and thereby for fully exploiting the vehicle's omnidirectionality.

The attitude error is defined as the rotation from the vehicle's current attitude to the desired attitude, i.e.

$$
\boldsymbol{q}_{\mathrm{err}}=\boldsymbol{q}_{\mathrm{des}} \otimes \boldsymbol{q}^{-1},
$$

where $\boldsymbol{q}^{-1}=\left(q_{0},-\tilde{\boldsymbol{q}}\right)$, resulting in the attitude error kinematics

$$
\dot{\boldsymbol{q}}_{\mathrm{err}}=\frac{1}{2}\left[\begin{array}{c}
0 \\
\boldsymbol{\omega}_{\mathrm{des}}
\end{array}\right] \otimes \boldsymbol{q}_{\mathrm{err}}-\frac{1}{2} \boldsymbol{q}_{\mathrm{err}} \otimes\left[\begin{array}{c}
0 \\
\boldsymbol{\omega}
\end{array}\right],
$$

where $\omega_{\text {des }}$ is the desired attitude trajectory's nominal angular velocity, i.e. the angular velocity that satisfies the attitude kinematics (50). Since the space of unit quaternions double covers the space of physical attitudes and each pair of antipodal unit quaternions $\pm \boldsymbol{q}$ represents the same physical attitude, an attitude control law capable of steering the vehicle to $\boldsymbol{q}_{\mathrm{err}}=( \pm 1,0,0,0)$ has to be employed in order to avoid the unwinding phenomena [35], i.e. that the vehicle unnecessarily performs a rotation larger than $\pi \mathrm{rad}$ to reach the desired attitude. In [34], the control law

$$
\boldsymbol{\omega}_{\mathrm{cmd}}=\frac{2}{\tau_{\mathrm{att}}} \operatorname{sgn}\left(q_{\mathrm{err}, 0}\right) \tilde{\boldsymbol{q}}_{\mathrm{err}}
$$

is shown to be globally asymptotically stable about the two equilibria $\boldsymbol{q}_{\mathrm{err}}=( \pm 1,0,0,0)$ for any stationary desired attitude $\boldsymbol{q}_{\mathrm{des}}$, where the sign of the scalar component of the attitude error is used as an indicator of which equilibrium is closer. By inserting (63) into the attitude error kinematics (62), it can be seen that for small deviations from the equilibria $\left(q_{\mathrm{err}, 0} \approx \pm 1,\left\|\tilde{\boldsymbol{q}}_{\mathrm{err}}\right\|_{2} \ll 1\right)$ the attitude error behaves like a first-order system with time constant $\tau_{\text {att }}$,

$$
\dot{\boldsymbol{q}}_{\mathrm{err}} \approx\left[\begin{array}{c}
0 \\
-\frac{1}{\tau_{\text {att }}} \tilde{\boldsymbol{q}}_{\mathrm{err}}
\end{array}\right] .
$$

In order to account for modelling errors and to not only be able to stabilize the multirotor vehicle to a desired attitude, but also track a desired attitude trajectory, the attitude control law (63) is extended by an integral term [36] with time constant $\tau_{\text {att }, \mathrm{i}}$ and a feed-forward term, resulting in

$$
\begin{aligned}
\boldsymbol{\omega}_{\mathrm{cmd}}= & k_{\mathrm{att}, \mathrm{p}} \operatorname{sgn}\left(q_{\mathrm{err}, 0}\right) \tilde{\boldsymbol{q}}_{\mathrm{err}} \\
& +k_{\mathrm{att}, \mathrm{i}} \int \operatorname{sgn}\left(q_{\mathrm{err}, 0}\right) \tilde{\boldsymbol{q}}_{\mathrm{err}} d t+\boldsymbol{R}\left(\boldsymbol{q}_{\mathrm{err}}\right)^{-1} \boldsymbol{\omega}_{\mathrm{des}},
\end{aligned}
$$

with the proportional and integral gains given by

$$
\begin{aligned}
& k_{\text {att }, \mathrm{p}}=\frac{2}{\tau_{\text {att }}}+\frac{2}{\tau_{\text {att }, \mathrm{i}}}, \\
& k_{\text {att }, \mathrm{i}}=\frac{2}{\tau_{\text {att }} \tau_{\text {att }, \mathrm{i}}} .
\end{aligned}
$$

The feed-forward term in (65) represents the desired angular velocity expressed in the vehicle's body-fixed frame $\mathcal{B}$ and cancels the first term of (62).

\section{Angular Velocity Control}

The attitude controller's commanded angular velocity is tracked by an inner control loop that is designed such that the components of the angular velocity $\omega$ track the angular velocity commands $\boldsymbol{\omega}_{\text {cmd }}$ like a linear time invariant first-order system with time constant $\tau_{\omega}$. Using feedback linearization to 
cancel the nonlinear terms in the attitude dynamics (54), the torque required for the angular velocity to achieve the desired first-order system response is found to be

$$
\boldsymbol{t}_{\mathrm{cmd}}=\frac{1}{\tau_{\omega}} \boldsymbol{J}\left(\boldsymbol{\omega}_{\mathrm{cmd}}-\boldsymbol{\omega}\right)+\boldsymbol{\omega} \times\left(\boldsymbol{J} \boldsymbol{\omega}+\sum_{i=1}^{8} J_{\mathrm{rot}} \Omega_{i} \boldsymbol{n}_{i}\right) .
$$

\section{E. Control Allocation}

The task of the control allocation is to compute rotor thrust commands $f_{\text {rot,cmd }}$ such that the commanded total thrust and torque $\boldsymbol{v}_{\mathrm{cmd}}=\left(\boldsymbol{f}_{\mathrm{cmd}}, \boldsymbol{t}_{\mathrm{cmd}}\right)$ are generated. Since the omnidirectional multirotor vehicle is over-actuated (it has eight rotors to generate a six dimensional thrust and torque), no unique map exists to allocate the commanded total thrust and torque $\boldsymbol{v}_{\text {cmd }}$ to rotor thrusts $f_{\text {rot,cmd }}$. We have thus decided to solve the control allocation problem by means of an optimization problem, where, in the long term, we seek to find the rotor thrust commands $f_{\text {rot,cmd }}$ that minimize the power consumption (see B),

$$
\sum_{i=1}^{8}\left|f_{\text {rot }, \mathrm{cmd}, i}\right|^{3 / 2}
$$

and in the short term, we seek to find the rotor thrust commands that minimize the difference to the current rotor thrusts $\boldsymbol{f}_{\text {rot }}$,

$$
\sum_{i=1}^{8}\left(f_{\text {rot }, \mathrm{cmd}, i}-f_{\mathrm{rot}, i}\right)^{2} .
$$

In the design of the cascaded control structure it is assumed that the underlying control loops can track set point changes without any dynamics or delay. However, the rotors cannot track commands arbitrarily fast in practice (see Section IV-B), especially when the direction of rotation of a rotor needs to be reversed [22]. The short-term objective can thus be interpreted as an attempt to take the rotor dynamics into account. By minimizing the difference between the commanded and the current rotor thrusts, the rotor thrust commands are in general easier to track and also reversing the direction of rotors is partially avoided (since this would correspond to a rotor thrust difference of at least $\left.2 f_{\min }\right)$. Nevertheless, it was experimentally found that the short-term objective only insufficiently avoids rotor reversals. In order to avoid that a rotor reverses its direction of rotation in rapid succession, which particularly violates the assumption that set point changes can be tracked without any dynamics or delay, we further employ a temporal hysteresis on the direction of rotation of the rotors. Let $\mathcal{F}_{\text {rot,hyst }} \subseteq \mathcal{F}_{\text {rot }}$ be the set of rotor thrusts that does not require any rotor to reverse its direction of rotation when it has already reversed its direction within the past $\tau_{\text {hyst }}$ timespan. The control allocation problem can then be stated as

$$
\begin{array}{ll}
\underset{\boldsymbol{f}_{\mathrm{rot}, \mathrm{cmd}}}{\operatorname{minimize}} & \sum_{i=1}^{8}(1-\epsilon)\left|f_{\text {rot }, \mathrm{cmd}, i}\right|^{3 / 2}+\epsilon\left(f_{\text {rot }, \mathrm{cmd}, i}-f_{\mathrm{rot}, i}\right)^{2} \\
\text { subject to } & \boldsymbol{v}_{\mathrm{cmd}}=\boldsymbol{B} \boldsymbol{f}_{\mathrm{rot}, \mathrm{cmd}}, \\
& \boldsymbol{f}_{\mathrm{rot}, \mathrm{cmd}} \in \mathcal{F}_{\text {rot }}^{\text {hyst }},
\end{array}
$$

where the scalar parameter $\epsilon \in[0,1]$ is used to weight the long-term versus the short-term objective and can be interpreted as a damping factor that determines the rate at which the rotor thrust commands converge to the minimum power solution. Due to the hysteresis constraint, it is possible that the control allocation problem (71) has no solution although the commanded total thrust and torque are attainable, i.e. $\boldsymbol{v}_{\text {cmd }} \in \mathcal{V}$. In this case, the time $\tau_{\text {hyst }}$ is decreased until a solution exists, with the limit case of $\mathcal{F}_{\text {rot,hyst }}=\mathcal{F}_{\text {rot }}$ for $\tau_{\text {hyst }}=0 \mathrm{~s}$.

Because the set of feasible rotor thrusts $\mathcal{F}_{\text {rot }}$ is not convex, solving the optimization problem (71) is computationally expensive and can typically not be done in real time on the low-cost microcontrollers found on multirotor vehicles. Instead of solving (71) directly, the structure of the control allocation problem, in particular of the null space of the thrust and torque map $\boldsymbol{B}$, is exploited, which yields a computationally cheap optimization problem that can easily be solved in real time even on low-cost microcontrollers. As shown in (19), all rotor thrusts that generate the commanded total thrust and torque can be written as the sum of any rotor thrusts that generate the commanded total thrust and torque and a multiple of the null vectors of $\boldsymbol{B}$,

$$
\boldsymbol{f}_{\mathrm{rot}, \mathrm{cmd}}=\boldsymbol{B}^{\dagger} \boldsymbol{v}_{\mathrm{cmd}}+\phi_{1} \boldsymbol{\eta}_{1}+\phi_{2} \boldsymbol{\eta}_{2}
$$

where $\boldsymbol{B}^{\dagger}$ is the pseudo-inverse of $\boldsymbol{B}$,

$$
\boldsymbol{B}^{\dagger}=\boldsymbol{B}^{T}\left(\boldsymbol{B} \boldsymbol{B}^{T}\right)^{-1} \text {. }
$$

In order to ensure that the commanded rotor thrusts are feasible, i.e. $\boldsymbol{f}_{\text {rot,cmd }} \in \mathcal{F}_{\text {rot }}$, the biases $\phi_{1}$ and $\phi_{2}$ need to be constrained to the sets $\Phi_{1}$ and $\Phi_{2}$, where

$$
\begin{aligned}
& \Phi_{1}=\left\{\phi_{1} \in \mathbb{R}\left|f_{\min } \mathbf{1} \preceq\right| \boldsymbol{P}_{1}\left(\boldsymbol{B}^{\dagger} \boldsymbol{v}_{\mathrm{cmd}}+\phi_{1} \boldsymbol{\eta}_{1}\right) \mid \preceq f_{\max } \mathbf{1}\right\}, \\
& \Phi_{2}=\left\{\phi_{2} \in \mathbb{R}\left|f_{\min } \mathbf{1} \preceq\right| \boldsymbol{P}_{2}\left(\boldsymbol{B}^{\dagger} \boldsymbol{v}_{\mathrm{cmd}}+\phi_{2} \boldsymbol{\eta}_{2}\right) \mid \preceq f_{\max } \mathbf{1}\right\},
\end{aligned}
$$

with $\boldsymbol{P}_{1}=\left(\boldsymbol{I}_{4 \times 4}, \mathbf{0}_{4 \times 4}\right)$ and $\boldsymbol{P}_{2}=\left(\mathbf{0}_{4 \times 4}, \boldsymbol{I}_{4 \times 4}\right)$. Because the null vectors $\boldsymbol{\eta}_{1}$ and $\boldsymbol{\eta}_{2}$ contain only ones and zeros, the sets $\Phi_{1}$ and $\Phi_{2}$ are straightforward to compute. Analogously to $\mathcal{F}_{\text {rot,hyst }}$, the sets $\Phi_{1 \text {,hyst }} \subseteq \Phi_{1}$ and $\Phi_{2, \text { hyst }} \subseteq \Phi_{2}$ are defined as the sets of feasible rotor thrust biases that do not require a rotor to reverse its direction of rotation if it has already reversed its direction of rotation recently. Inserting (72) into (71) then reduces the original control allocation problem with eight optimization variables to two independent optimization problems with each having only a single optimization variable, the bias $\phi_{1}$ and the bias $\phi_{2}$, respectively. The optimization problem for $\phi_{1}$ reads as

$$
\begin{aligned}
\underset{\phi_{1}}{\operatorname{minimize}} \quad \sum_{i=1}^{4}(1-\epsilon) \mid & \hat{f}_{\mathrm{rot}, \mathrm{cmd}, i}+\left.\phi_{1}\right|^{3 / 2} \\
& +\epsilon\left(\hat{f}_{\mathrm{rot}, \mathrm{cmd}, i}+\phi_{1}-f_{\mathrm{rot}, i}\right)^{2}
\end{aligned}
$$

subject to $\phi_{1} \in \Phi_{1, \text { hyst }}$,

where $\hat{\boldsymbol{f}}_{\text {rot,cmd }}=\boldsymbol{B}^{\dagger} \boldsymbol{v}_{\mathrm{cmd}}$. The optimization problem for $\phi_{2}$ can be written analogously to (76), but will not be discussed in the following due to symmetry. Since the cost function of (76) is convex, the optimal bias $\phi_{1}^{*}$ can be computed by 
first calculating the bias $\tilde{\phi}_{1}^{*}$ that minimizes the cost in the unconstrained case. Then, if $\tilde{\phi}_{1}^{*} \in \Phi_{1 \text {,hyst }}$, the optimal bias is given by $\phi_{1}^{*}=\tilde{\phi}_{1}^{*}$, and otherwise the optimal bias is found at the boundaries of $\Phi_{1 \text {,hyst }}$. In practice, the $\tilde{\phi}_{1}^{*}$ is computed by employing bisection ${ }^{6}$ to find the bias where the derivative of the objective function with respect to $\phi_{1}$ is zero, i.e.

$$
\begin{aligned}
\sum_{i=1}^{4} \frac{3}{2}(1-\epsilon)\left|\hat{f}_{\text {rot }, \mathrm{cmd}, i}+\phi_{1}\right|^{1 / 2} & \\
& +2 \epsilon\left(\hat{f}_{\mathrm{rot}, \mathrm{cmd}, i}+\phi_{1}-f_{\mathrm{rot}}, i\right) \stackrel{!}{=} 0 .
\end{aligned}
$$

One issue that can arise in the constrained case, i.e. when $\phi_{1} \in \Phi_{1 \text {,hyst }}$, is that a lot of power is wasted due to the second term of the objective function that penalizes large differences in the rotor thrusts and prevents $\phi_{1}^{*}$ to jump to a solution that consumes less power. This issue can be circumvented by only allowing solutions that consume less power than in the unconstrained case (if one exists), i.e. by adding the constraint

$$
\sum_{i=1}^{4}\left|\hat{f}_{\mathrm{rot}, \mathrm{cmd}, i}+\phi_{1}\right|^{3 / 2} \leq \sum_{i=1}^{4}\left|\hat{f}_{\mathrm{rot}, \mathrm{cmd}, i}+\tilde{\phi}_{1}^{*}\right|^{3 / 2}
$$

to the optimization problem (76).

Once the optimal biases $\phi_{1}^{*}$ and $\phi_{2}^{*}$ are computed, the individual rotor thrust commands are found by evaluating (72) and are subsequently converted into rotor angular velocity commands using the rotor thrust model (42),

$$
\Omega_{\mathrm{cmd}, i}=\operatorname{sgn}\left(f_{\mathrm{rot}, \mathrm{cmd}, i}\right) \sqrt{\frac{\left|f_{\mathrm{rot}, \mathrm{cmd}, i}\right|}{c_{f}}} .
$$

Fig. 17 shows the behaviour of the control allocation strategy for varying thrust and torque commands including reversing the rotors.

\section{F. Motor Control}

For traditional multirotor vehicles where all rotor disks are aligned in a single plane, the direction of the total thrust is independent of the motors' angular velocities. However, due to the non-planar rotor configuration (11)-(12), the direction of the total thrust and torque of the proposed omni-directional vehicle depends on the angular velocity of each motor, and therefore, accurate control thereof is of fundamental importance. A feedback linearizing controller is employed such that angular velocity commands are tracked in the fashion of a linear, time-invariant, first-order system with time constant $\tau_{\text {mot }}$,

$$
\dot{\Omega}_{i}=\frac{1}{\tau_{\mathrm{mot}}}\left(\Omega_{\mathrm{cmd}, i}-\Omega_{i}\right) .
$$

In order to be less prone to variations in the motor gain $R J_{\text {rot }} / k_{e}$ and steady-state voltage $\bar{u}\left(\Omega_{i}\right)$ between different motors, the controller is augmented with an integral term with

\footnotetext{
${ }^{6}$ Bisection is used to find the root of (77) because of its simplicity and because the derivative of (77) with respect to $\phi_{1}$ is not defined, therefore rendering other commonly used methods that rely on the function's derivative infeasible.
}

TABLE IV: Control parameters.

\begin{tabular}{cll}
\hline Symbol & Description & Value \\
\hline$\tau_{\text {pos }}$ & Position control time constant & $0.325 \mathrm{~s}$ \\
$\tau_{\text {pos,i }}$ & Position control integral time constant & $1.33 \mathrm{~s}$ \\
$\zeta_{\text {pos }}$ & Position control damping ratio & 1.0 \\
$\tau_{\text {att }}$ & Attitude control time constant & $0.39 \mathrm{~s}$ \\
$\tau_{\text {att,i }}$ & Attitude control integral time constant & $1.5 \mathrm{~s}$ \\
$\tau_{\omega}$ & Angular velocity control time constant & $0.044 \mathrm{~s}$ \\
& Control allocation short vs. long-term & 0.95 \\
$\tau_{\text {hyst }}$ & objective weight & \\
$\tau_{\text {mot }}$ & Memporal hysteresis on rotation direction & $0.75 \mathrm{~s}$ \\
$\tau_{\text {mot,int }}$ & Motor control integral time constant & $0.032 \mathrm{~s}$ \\
& & $0.199 \mathrm{~s}$
\end{tabular}

time constant $\tau_{\text {mot,int }}$. The voltage command is then found using (47),

$$
\begin{aligned}
u_{\mathrm{cmd}, i}=\frac{R J_{\mathrm{rot}}}{k_{e}}( & k_{\mathrm{mot}, \mathrm{p}}\left(\Omega_{\mathrm{cmd}, i}-\Omega_{i}\right) \\
& \left.+k_{\mathrm{mot}, \mathrm{i}} \int\left(\Omega_{\mathrm{cmd}, i}-\Omega_{i}\right) d t\right)+\bar{u}\left(\Omega_{i}\right),
\end{aligned}
$$

where the proportional and integral gains $k_{\mathrm{mot}, \mathrm{p}}$ and $k_{\mathrm{mot}, \mathrm{i}}$ are given by

$$
\begin{aligned}
k_{\mathrm{mot}, \mathrm{p}} & =\frac{\tau_{\mathrm{mot}, \text { int }}}{\tau_{\mathrm{mot}}\left(\tau_{\mathrm{mot}, \mathrm{int}}-\tau_{\mathrm{mot}}\right)}, \\
k_{\mathrm{mot}, \mathrm{i}} & =\frac{1}{\tau_{\mathrm{mot}}\left(\tau_{\mathrm{mot}, \mathrm{int}}-\tau_{\mathrm{mot}}\right)} .
\end{aligned}
$$

\section{G. Discussion}

All controllers are shaped to respond to commands in the fashion of linear time-invariant first or second-order systems with the tuning parameters being time constants and damping ratios. The time constants and damping ratios of each control loop were manually tuned on the real system and are listed in Table IV.

Note that the effects of actuator saturation are not considered in the proposed control design and special attention needs to be paid when implementing the controllers with integral action to avoid integrator windup [33], [36]. Because devising control strategies that guarantee stability in the presence of actuator saturations is a difficult problem, in particular when not just stabilizing a system but tracking trajectories, it is considered to be beyond the scope of this paper and left for future work. The proposed control strategy only yields the desired first and second-order system responses when the errors are small. Total thrust and torque commands $\boldsymbol{v}_{\mathrm{cmd}}$ that lie outside the set of attainable total thrusts and torque $\mathcal{V}$ are currently handled by projecting them onto the boundary of the set of attainable total thrusts and torques while preserving their direction. This can be done in a computationally efficient manner by downscaling the total thrust and torque command until the differences between the largest and smallest rotor thrust of $\hat{\boldsymbol{f}}_{\text {rot,cmd }}=\boldsymbol{B}^{\dagger} \boldsymbol{v}_{\text {cmd }}$ for the rotors 1-4 and 5-8 are smaller than $2 f_{\max }-4 f_{\min }$ (see Section II-E), and has shown satisfactory results in practice. 


\section{RESULTS}

This section presents experimental flight tests that were carried out in order to validate the vehicle design and control strategy as well as to demonstrate the capabilities of the omnidirectional multirotor vehicle.

A video of the vehicle performing various maneuvers is available at https://youtu.be/sIi 80 LMLJSY. The vehicle has further been showcased in front of a live audience during Raffaello D'Andrea's talk at the TED conference 2016, available at https://youtu.be/RCXGpEmFbOw.

\section{A. Experimental Setup}

All experiments following were carried out in the Flying Machine Arena [37], an indoor test bed for aerial vehicles at ETH Zurich. The Flying Machine Arena is equipped with a motion capture system that measures the vehicle's pose at a rate of $200 \mathrm{~Hz}$. The pose measurements are processed on a desktop computer by a Luenberger observer that estimates the vehicle's position, velocity and attitude. Based on these estimates, thrust and angular velocity commands are computed by the off-board position and attitude controller at a rate of $50 \mathrm{~Hz}$ and subsequently sent to the vehicle through a lowlatency radio link. The closed-loop latency between observing the vehicle's pose and receiving commands on board the vehicle was identified to be $39 \mathrm{~ms}$ and is compensated by running the off-board controllers with state estimates that are predicted forward in time by the closed-loop latency. The offboard angular velocity controller and control allocation run at a rate of $500 \mathrm{~Hz}$ and the motor controllers run at $1 \mathrm{kHz}$.

\section{B. Aerodynamic Interference}

In the model of the rotor thrust (42) and torque (44), it was assumed that the air surrounding the rotors is stationary and aerodynamic effects such as interference between the rotors were neglected. In order to verify this assumption and characterize the effects of aerodynamic interference, thrust and torque measurements of the vehicle during flight are taken and compared with the thrust and torque predicted by the total thrust and torque model (2) and (3) based on the measured rotors' angular velocities. In order to obtain accurate thrust and torque measurements, the vehicle is commanded to hover at random attitudes while carrying weights of varying masses between $0.028 \mathrm{~kg}$ and $0.126 \mathrm{~kg}$ mounted on the vehicle at different positions approximately $0.46 \mathrm{~m}$ off the vehicle's center of mass. The thrust and torque generated by the rotors are then inferred from the vehicle's attitude and the gravitational acceleration that acts on the vehicle. A total of 2036 measurements at uniformly sampled random attitudes were taken, and the RMSE of the Euclidean norm of the thrust and torque residuals were computed to be $1.73 \mathrm{~N}$ and $0.235 \mathrm{~N} \mathrm{~m}$, respectively.

It was found that the rotors generate significantly less thrust and torque than that measured on a load cell for a single rotor due to the fact that the thrust and torque efficacy of a rotor decreases with increasing speed of the incoming air flow. By

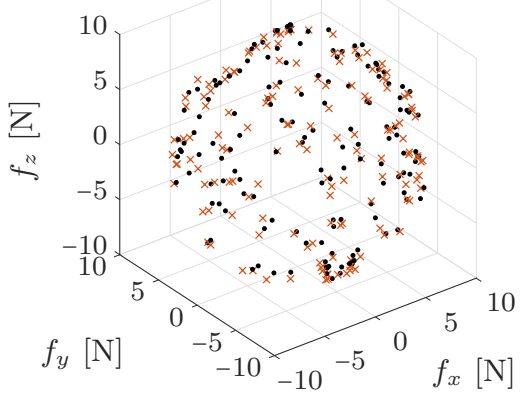

Fig. 13: 150 samples of total thrust generated by the vehicle when hovering at different attitudes. The crosses represent the total thrust inferred from the pose measurements and the dots represent the predicted thrust based on the rotor angular velocities and the adjusted thrust and torque coefficients.

minimizing the RMSE of the total thrust and torque residuals ${ }^{7}$, the decrease in efficacy was identified to be $14.2 \%$ for the thrust and $19.5 \%$ for the torque, resulting in RMSE of $0.75 \mathrm{~N}$ and $0.192 \mathrm{Nm}$, respectively. Fig. 13 shows 150 samples of the measured total thrust and its predicted total thrust using the fitted thrust efficacy. Due to the highly symmetric rotor configuration, it was assumed that the decrease in efficacy is equal for each rotor. Although more complex models could be fitted, assuming an equal decrease for each rotor was found to work well in practice and furthermore allows for a simple computation of the required rotor angular velocity for a given total thrust and torque command.

In the following experiments, the rotor angular velocity commands are compensated for the decrease in the thrust and torque efficacy.

\section{Decoupled Position and Attitude Dynamics}

Using the proposed control strategy, the vehicle can fly arbitrary position and attitude paths. As an example, the vehicle is commanded to track a figure of eight at a speed of approximately $1 \mathrm{~m} / \mathrm{s}$ in the inertial $x z$-plane while simultaneously rotating about the body-fixed axis $1 / \sqrt{6}(\sqrt{2}, \sqrt{3},-1)$ at a rate of $0.4 \mathrm{rad} / \mathrm{s}$. The figure of eight consists of two circle segments with a radius of $1.2 \mathrm{~m}$ that are connected by splines, where the order of the splines is chosen such that the nominal thrust and torque commands are continuous. Fig. 14 shows a time lapse of the vehicle tracking the maneuver, and in Fig. 15 and Fig. 16, the position and attitude errors as well as the corresponding rotor thrust commands for three executions of the maneuver are illustrated. The position and attitude tracking error appear to correlate, indicating that the control strategy is not able to fully decouple the vehicle's position and attitude dynamics. Furthermore, the errors appear to be repetitive, which could be due to unmodelled aerodynamic effects. The

\footnotetext{
${ }^{7}$ The torque was normalized by the inverse of rotor distance from the vehicle's center of mass such that a rotor thrust of $1 \mathrm{~N}$ of generates a torque of $1 \mathrm{Nm}$.
} 
peaks in the attitude error, for example at around $t=3 \mathrm{~s}$, are caused by one of the rotors reversing its direction of rotation, after which the vehicle sometimes struggles to maintain its attitude.

\section{Control Allocation}

The effect of the control allocation strategy becomes most apparent when the commanded total thrust or torque changes substantially, such as when the vehicle executes large attitude changes. The two upper plots of Fig. 17 depict the commanded total thrust and torque of the vehicle when rotating at a fixed position about its body-fixed $x$-axis at a rate of $0.6 \mathrm{rad} / \mathrm{s}$ for $\pi \mathrm{rad}$. The two lower plots depict the corresponding rotor thrust commands of rotors 1-4 and their bias $\phi_{1}^{*}$, respectively. It can be seen that the control allocation attempts to bias the rotor thrusts in order to prevent the rotors from changing their direction of rotation (for example shortly $t=4 \mathrm{~s}$ ). If the bias becomes too large, i.e. the total thrust and torque is generated inefficiently, the bias jumps by $\pm 2 f_{\min }$ such that the rotor that previously generated a thrust of $f_{\text {min }}$ now generates a thrust of $-f_{\min }$ or vice versa. Due to the second term in the control allocation objective function (71) that penalizes large differences in the rotor thrusts, the bias does not jump to the minimum power solution even if it would be feasible, but gradually approaches the minimum power solution. Sometimes after the direction of rotation of a rotor is reversed, the vehicle's attitude and consequently the commanded total torque begin to oscillate, as can be seen at $t=2.3 \mathrm{~s}$.

\section{CONCLUSION}

This paper presented the mechatronic design of an omnidirectional multirotor vehicle. Based on a static thrust and torque analysis, an octorotor configuration was derived that maximizes the vehicle's agility while rendering its characteristics almost rotationally invariant and its set of attainable thrusts and torques was analyzed. A prototype vehicle with the derived rotor configuration was built using reversible fixed-pitch rotors that enable the vehicle to independently generate thrusts and torques in any direction. A simplified model of the vehicle dynamics was introduced and a cascaded control strategy was presented that allows the vehicle to simultaneously track a desired position and attitude trajectory. Experimental flight tests were conducted to validate the feasibility of the vehicle design and the proposed control strategy.

The ability of the vehicle to independently control its thrust and torque in any direction facilitates novel flight maneuvers and enables the vehicle to, for example, fly in confined spaces or take off at arbitrary attitudes. However, these benefits come at the expense of increased vehicle mass and reduced hover efficiency with respect to traditional multirotor vehicles. Any application will have to consider the tradeoff between increased maneuverability and reduced payload, range and flight duration.

When deriving a model for the thrust and torque generated by the rotors, the aerodynamic interference between the rotors was neglected and its effect on the rotors was characterized in a later step, however, only for near hover conditions.
Similar simplifying assumptions were made in the design of the control strategy, where, for example, the effects of rotor thrust saturations were neglected and it was assumed that the errors from the desired trajectories are small. The applicability of both the vehicle design and control strategy at high speeds or under large wind disturbances thus remains to be proven in future work. A possibility to circumvent the problem of deriving an accurate model for the rotor thrust and torque despite significant interference between rotors could be to directly control the aerodynamic power of a rotor instead of its angular velocity [38], which was shown to be more robust to difficult to model aerodynamic effects such as rotor interference, but requires the ability to accurately measure the power consumed by each rotor.

The experimental results demonstrated the vehicle's omnidirectionality, but have also revealed that the vehicle struggles to maintain its attitude when one of the rotors is reversing its direction of rotation. When a rotor is reversed, a large reaction torque acts on the vehicle due to the change in the rotor's angular momentum. In the proposed control strategy, this reaction torque is neglected and treated as a disturbance. Possible future work could therefore be to identify these disturbances using, for example, iterative learning and consider them in a feed-forward term. Another reason for the vehicle's struggle to maintain its attitude when a rotor's direction of rotation is reversed lies in the control allocation strategy, where it is assumed that the rotors can track the angular velocity commands sufficiently fast. However, when a rotor's direction of rotation is reversed, it is operated in open-loop for a short period of time and its angular velocity cannot be controlled at all. The control allocation strategy should therefore only allocate the desired total thrust and torque to the rotors that are controllable. Similarly, this strategy could also be employed in order to maintain safe flight in case of a rotor failure.

Apart from improving the control strategy and verifying the applicability of the vehicle design and control strategy at high speeds, possible future work also includes the use of the proposed multirotor vehicle for aerial physical interaction. The vehicle's ability to independently control its thrust and torque in any direction is envisioned to be advantageous to cope with large external disturbances that typically occur in such scenarios. Furthermore, the vehicle's omni-directionality could be exploited to manipulate objects with a simple gripper rigidly attached to the vehicle instead of using a complex multi-degree-of-freedom robot arm.

\section{APPENDIX A}

MaXimum Minimum Singular Value of the Thrust AND TORQUE MAP

In this appendix, the theoretical maximum of the smallest singular value of the simplified thrust and torque map $\tilde{\boldsymbol{B}}$ is derived.

The singular values of any matrix $\boldsymbol{A} \in \mathbb{R}^{M \times N}$, with $M \leq N$, are given by the square root of the eigenvalues of $A A^{T}$

$$
\sigma_{j}(\boldsymbol{A})=\sqrt{\lambda_{j}\left(\boldsymbol{A} \boldsymbol{A}^{T}\right)}, \quad j=1, \ldots, M,
$$




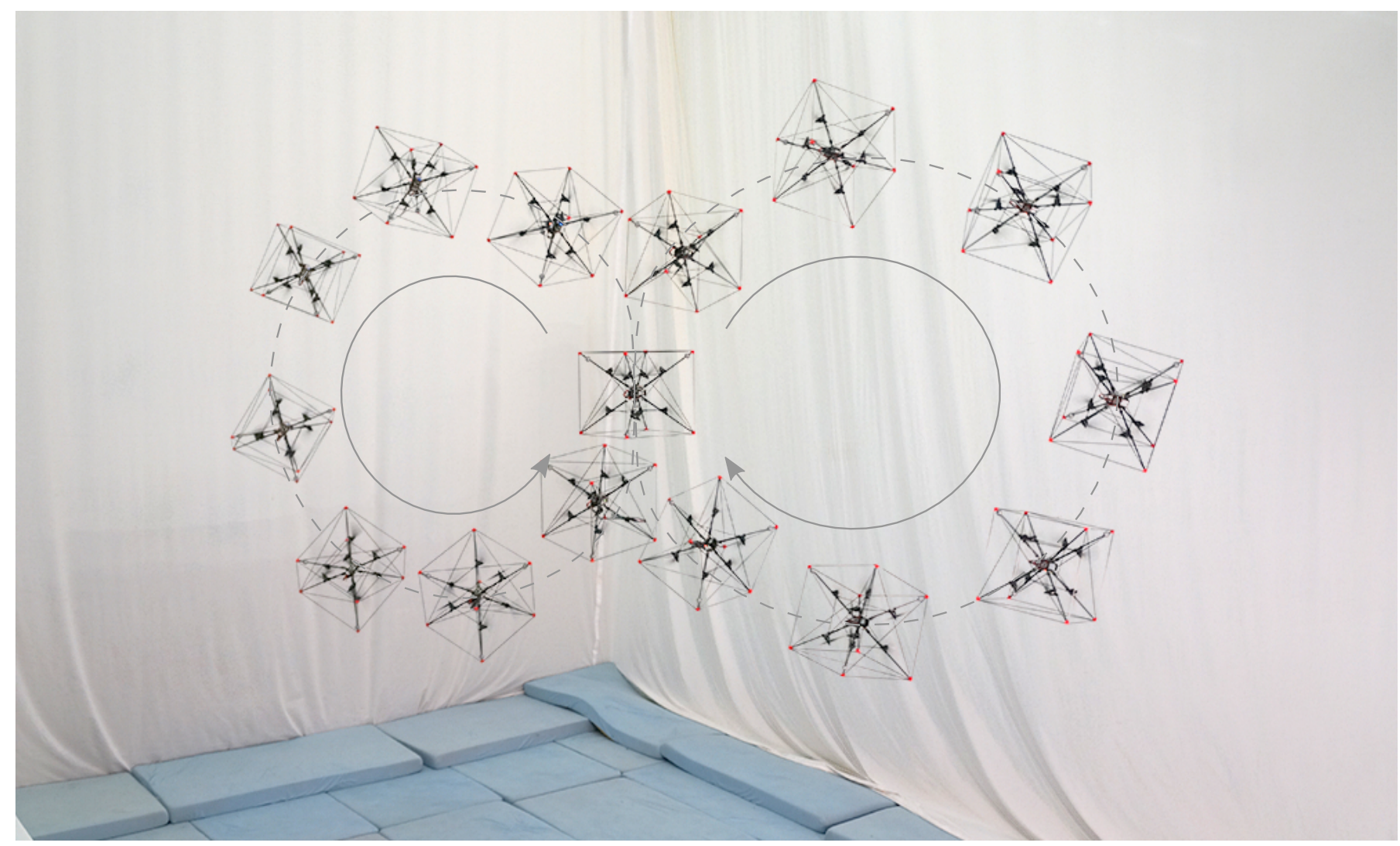

Fig. 14: Time lapse of the vehicle tracking a figure of eight at a speed of approximately $1 \mathrm{~m} / \mathrm{s}$ while simultaneously rotating about a body-fixed axis. The vehicle starts in the center at $t=0 \mathrm{~s}$ and then moves towards the right-hand side. Snapshots of the vehicle are taken once every full second until the full figure of eight is completed at $t=15.71 \mathrm{~s}$. The dashed line indicates the reference position.

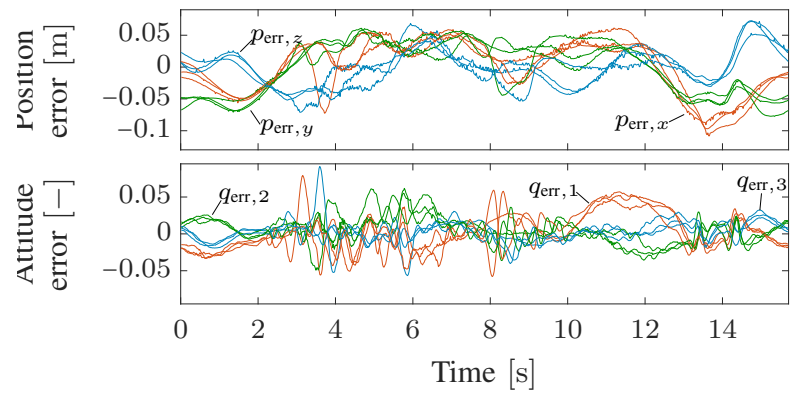

Fig. 15: The position and attitude tracking errors from three executions of the figure of eight maneuver. The top plot shows the position errors along each axis of the inertial frame $\mathcal{I}$, and the bottom plot depicts the components of the attitude error $\boldsymbol{q}_{\text {err }}$. The RMSE of the position and the attitude are $0.064 \mathrm{~m}$ and $0.069 \mathrm{rad}$, respectively.

Maximizing the smallest singular value $\sigma_{\min }$ is therefore equivalent to maximizing the smallest eigenvalue $\lambda_{\min }$. The sum of all eigenvalues of a matrix is equal to its trace, i.e.

$$
\sum_{j=1}^{M} \lambda_{j}\left(\boldsymbol{A} \boldsymbol{A}^{T}\right)=\operatorname{trace}\left(\boldsymbol{A} \boldsymbol{A}^{T}\right) .
$$

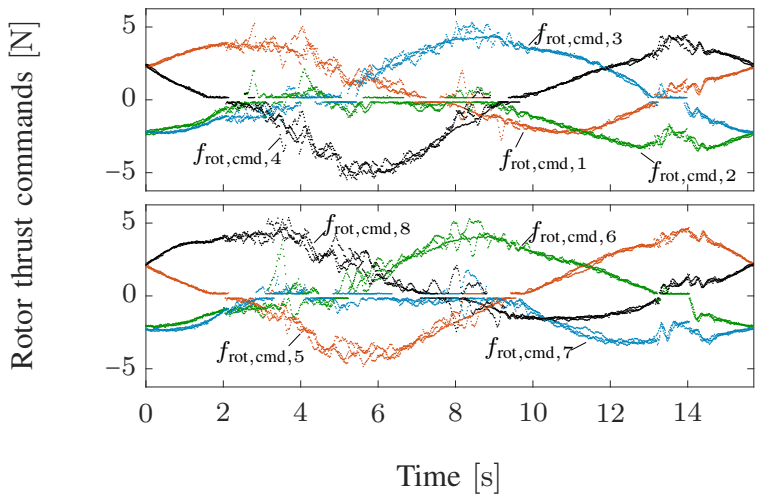

Fig. 16: The rotor thrust commands corresponding to the three executions of the figure of eight maneuver shown in Fig. 15. The top plot depicts the rotor thrust commands of rotors 1-4 and the bottom plot of rotors 5-8. For the sake of legibility, only every tenth command is shown.

Let $\boldsymbol{a}_{i}$ denote the $i$-th column of $\boldsymbol{A}$. The trace of $\boldsymbol{A} \boldsymbol{A}^{T}$ is 


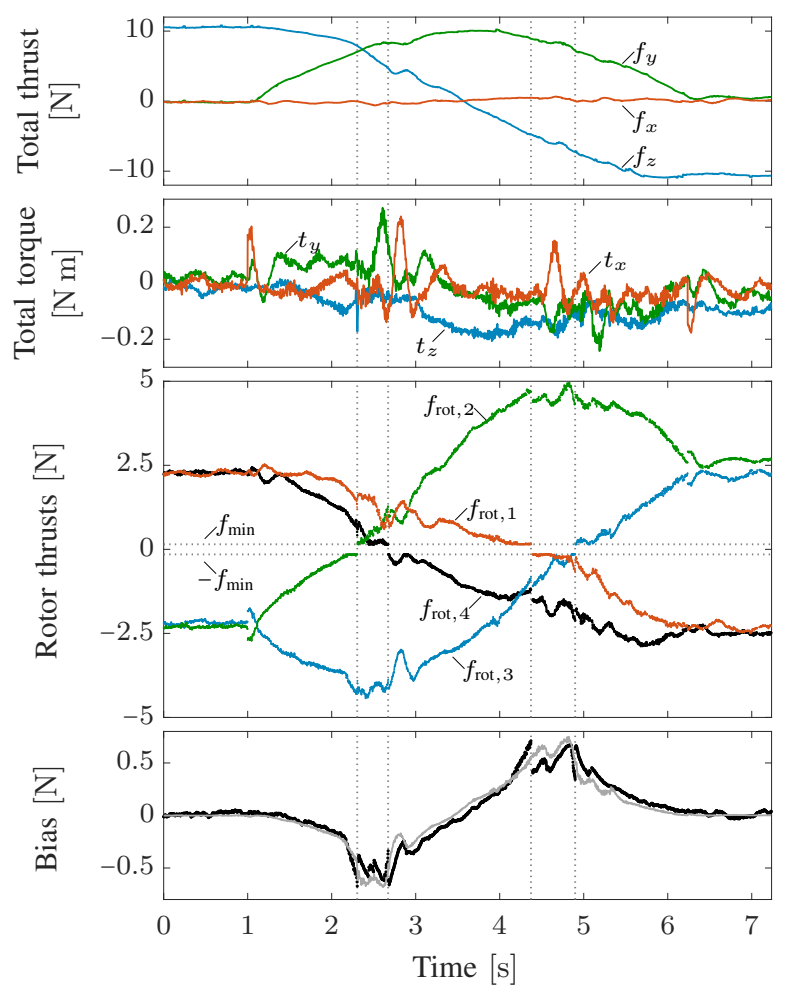

Fig. 17: The vehicle's total thrust and torque commands and corresponding rotor thrusts and bias of rotors 1-4 when performing a rotation about its body-fixed $x$-axis for $\pi \mathrm{rad}$. The rotation starts at $t=1 \mathrm{~s}$. The vertical dotted lines indicate the time instants when one of the rotors is reversing its direction of rotation. The black line in the bottom plot represents the applied bias of rotors 1-4 whereas the gray line represents the power optimal bias.

then straightforward to compute and is given by

$$
\begin{aligned}
\operatorname{trace}\left(\boldsymbol{A} \boldsymbol{A}^{T}\right) & =\operatorname{trace}\left(\boldsymbol{A}^{T} \boldsymbol{A}\right) . \\
& =\sum_{i=1}^{N} \boldsymbol{a}_{i}^{T} \boldsymbol{a}_{i} .
\end{aligned}
$$

Evaluating (87) for the simplified thrust and torque map $\tilde{\boldsymbol{B}}$ whose columns are given by $\boldsymbol{b}_{i}=\left(\boldsymbol{n}_{i}, \boldsymbol{p}_{i} \times \boldsymbol{n}_{i}\right)$ yields

$$
\begin{aligned}
\operatorname{trace}\left(\tilde{\boldsymbol{B}} \tilde{\boldsymbol{B}}^{T}\right) & =\sum_{i=1}^{N} \boldsymbol{b}_{i}^{T} \boldsymbol{b}_{i}, \\
& =\sum_{i=1}^{N}\left(\boldsymbol{n}_{i}^{T} \boldsymbol{n}_{i}+\left(\boldsymbol{p}_{i} \times \boldsymbol{n}_{i}\right)^{T}\left(\boldsymbol{p}_{i} \times \boldsymbol{n}_{i}\right)\right) \\
& \leq 2 N,
\end{aligned}
$$

where it was used that the rotor positions in the vehicle design are normalized to unit length, i.e. $\left\|\boldsymbol{p}_{i}\right\|_{2}=1$. Since there is an upper bound on the sum of the eigenvalues, the smallest eigenvalue is maximized if every eigenvalue is equally large, i.e.

$$
\lambda_{j}=\frac{2 N}{6}, \quad j=1, \ldots, 6
$$

which implies that smallest singular value of $\tilde{\boldsymbol{B}}$ can take at most the value $\sqrt{N / 3}$.

\section{APPENDiX B \\ ROTOR POWER CONSUMPTION}

In this appendix, the power required by the $i$-th rotor to generate a thrust $f_{\text {rot }, i}$ is identified. For the sake of readability, the subscript $i$ is omitted in the remainder of this appendix.

The power consumed by an individual rotor is determined by the voltage $u$ that is applied to the motor and the current $i$ that runs through its phase windings,

$$
P=u i .
$$

By inserting the motor's electrical dynamics (45) and mechanical dynamics (46) into (92), the static power required for a rotor to rotate at an angular velocity $\Omega$ is found to be

$$
P=\frac{R t_{\text {load }}(\Omega)^{2}}{k_{e}}+\Omega t_{\text {load }}(\Omega) .
$$

The motor load torque $t_{\text {load }}(\Omega)$ is mainly due to the aerodynamic drag of the rotor, which is proportional to the square of the angular velocity, and to a smaller extent due to friction and viscous damping. It therefore follows that the first term in (93), which represents the electrical power loss of the rotor, is characterized by a fourth-order polynomial in angular velocity and that the second term in (93), which represents the mechanical power, is characterized by a thirdorder polynomial in angular velocity. Experimental results of the power consumption of a single rotor are shown in Fig. 18. It can be seen that mechanical power dominates the power consumption and that although the electrical power losses are significant at high angular velocities, the power consumption can be well approximated by a term that is proportional to the third power of the angular velocity

$$
P \propto \Omega^{3} .
$$

Since the thrust magnitude is proportional to the angular velocity squared (42), it follows that the power required for a rotor to generate a rotor thrust of magnitude $\left|f_{\text {rot }}\right|$ is approximately given by

$$
P \propto\left|f_{\text {rot }}\right|^{3 / 2} .
$$

\section{ACKNOWLEDGEMENT}

This work is supported by and builds upon prior contributions by numerous collaborators in the Flying Machine Arena. A list of past and present participants of the project is available at http://flyingmachinearena.org. A special mention goes to Alex Wilkinson for his contributions to the mechanical design and to Max Kriegleder and Marc-Andrè Corzillius for assisting with the development of the electronics.

This research was supported by the Swiss National Science Foundation (SNSF). 


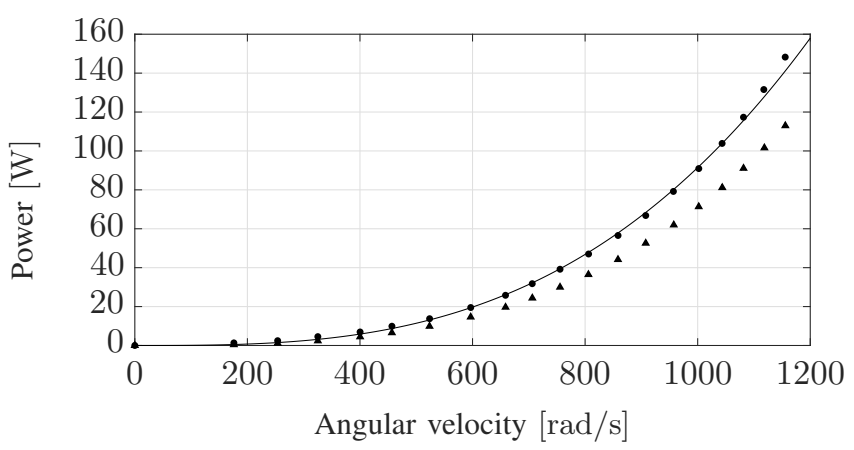

Fig. 18: The dots depict the experimentally determined power required for the rotor to spin at a given angular velocity and the triangles represent the measured mechanical power output. The required power can be approximated by a function that is proportional to the third power of the angular velocity (solid line) with a RMSE of $0.401 \mathrm{~W}$. Due to symmetry, only positive angular velocities are shown.

\section{REFERENCES}

[1] M. Mazur, A. Wisniewski, and J. McMillan, "Clarity from above," 2016. [Online]. Available: https://www.pwc.pl/pl/pdf/clarityfrom-above-pwc.pdf

[2] R. Mahony, V. Kumar, and P. Corke, "Multirotor aerial vehicles: Modeling, estimation, and control of quadrotor," IEEE Robotics Automation Magazine, vol. 19, no. 3, pp. 20-32, Sept 2012.

[3] G. Jiang and R. Voyles, "A nonparallel hexrotor uav with faster response to disturbances for precision position keeping," in IEEE International Symposium on Safety, Security, and Rescue Robotics (SSRR), Oct 2014, pp. 1-5.

[4] D. Mellinger, Q. Lindsey, M. Shomin, and V. Kumar, "Design, modeling, estimation and control for aerial grasping and manipulation," in IEEE/RSJ International Conference on Intelligent Robots and Systems (IROS), Sept 2011, pp. 2668-2673.

[5] S. Kim, S. Choi, and H. J. Kim, "Aerial manipulation using a quadrotor with a two dof robotic arm," in IEEE/RSJ International Conference on Intelligent Robots and Systems (IROS), Nov 2013, pp. 4990-4995.

[6] F. Augugliaro, A. Mirjan, F. Gramazio, M. Kohler, and R. D'Andrea, "Building tensile structures with flying machines," in IEEE/RSJ International Conference on Intelligent Robots and Systems (IROS), Nov 2013, pp. 3487-3492.

[7] F. Augugliaro and R. D'Andrea, "Admittance control for physical human-quadrocopter interaction," in European Control Conference (ECC), July 2013, pp. 1805-1810.

[8] K. Nitta, K. Higuchi, and J. Rekimoto, "HoverBall: Augmented sports with a flying ball," in Proceedings of the 5th Augmented Human International Conference, ser. AH '14. New York, NY, USA: ACM, 2014, pp. 13:1-13:4.

[9] A. E. Jimenez-Cano, J. Martin, G. Heredia, A. Ollero, and R. Cano, "Control of an aerial robot with multi-link arm for assembly tasks," in IEEE International Conference on Robotics and Automation (ICRA), May 2013, pp. 4916-4921.

[10] D. Wuthier, D. Kominiak, C. Kanellakis, G. Andrikopoulos, M. Fumagalli, G. Schipper, and G. Nikolakopoulos, "On the design, modeling and control of a novel compact aerial manipulator," in 24th Mediterranean Conference on Control and Automation (MED), June 2016, pp. 665-670.

[11] B. Crowther, A. Lanzon, M. Maya-Gonzalez, and D. Langkamp, "Kinematic analysis and control design for a nonplanar multirotor vehicle," Journal of Guidance, Control, and Dynamics, vol. 34, no. 4, pp. 11571171, Jul. 2011.

[12] G. Jiang and R. Voyles, "Hexrotor uav platform enabling dextrous interaction with structures-flight test," in IEEE International Symposium on Safety, Security, and Rescue Robotics (SSRR), Oct 2013, pp. 1-6.

[13] E. Kaufman, K. Caldwell, D. Lee, and T. Lee, "Design and development of a free-floating hexrotor uav for 6-dof maneuvers," in IEEE Aerospace Conference, March 2014, pp. 1-10.
[14] S. Rajappa, M. Ryll, H. H. Blthoff, and A. Franchi, "Modeling, control and design optimization for a fully-actuated hexarotor aerial vehicle with tilted propellers," in IEEE International Conference on Robotics and Automation (ICRA), May 2015, pp. 4006-4013.

[15] A. Nikou, G. C. Gavridis, and K. J. Kyriakopoulos, "Mechanical design, modelling and control of a novel aerial manipulator," in IEEE International Conference on Robotics and Automation (ICRA), May 2015, pp. 4698-4703.

[16] S. Park, J. Her, J. Kim, and D. Lee, "Design, modeling and control of omni-directional aerial robot," in IEEE/RSJ International Conference on Intelligent Robots and Systems (IROS), Oct 2016, pp. 1570-1575.

[17] M. Ryll, H. H. Bülthoff, and P. R. Giordano, "Modeling and control of a quadrotor uav with tilting propellers," in IEEE International Conference on Robotics and Automation (ICRA), May 2012, pp. 4606-4613.

[18] P. Segui-Gasco, Y. Al-Rihani, H. S. Shin, and A. Savvaris, "A novel actuation concept for a multi rotor uav," in International Conference on Unmanned Aircraft Systems (ICUAS), May 2013, pp. 373-382.

[19] Y. Long and D. J. Cappelleri, Omnicopter: A Novel Overactuated Micro Aerial Vehicle. Heidelberg: Springer International Publishing, 2013, pp. 215-226.

[20] M. Ryll, D. Bicego, and A. Franchi, "Modeling and control of fasthex: A fully-actuated by synchronized-tilting hexarotor," in IEEE/RSJ International Conference on Intelligent Robots and Systems (IROS), Oct 2016, pp. 1689-1694.

[21] D. Brescianini and R. D'Andrea, "Design, modeling and control of an omni-directional aerial vehicle," in IEEE International Conference on Robotics and Automation (ICRA), May 2016, pp. 3261-3266.

[22] M. Cutler and J. P. How, "Analysis and control of a variable-pitch quadrotor for agile flight," Journal of Dynamic Systems, Measurement, and Control, vol. 137, no. 10, pp. 101 002-1-101 002-14, Jul. 2015.

[23] R. W. Deters, G. K. Ananda Krishnan, and M. S. Selig, "Reynolds number effects on the performance of small-scale propellers," in 32nd AIAA Applied Aerodynamics Conference. American Institute of Aeronautics and Astronautics, Jun. 2014.

[24] P. K. Aravind, "A comment on the moment of inertia of symmetrical solids," American Journal of Physics, vol. 60, no. 8, pp. 754-755, 1992.

[25] W. C. Durham, "Attainable moments for the constrained control allocation problem," Journal of Guidance, Control, and Dynamics, vol. 17, no. 6, pp. 1371-1373, Nov. 1994.

[26] S. Kirby, "tgy - open source firmware for atmega-based brushless escs," 2017, [Online; accessed 11-December-2017]. [Online]. Available: http://0x.ca/tgy/

[27] Contributors. of nuttx.org, "Nuttx documentation - nuttx realtime operating system," 2017, [Online; accessed 11-December-2017]. [Online]. Available: http://nuttx.org/doku.php?id=documentation

[28] B. W. McCormick, Aerodynamics, aeronautics, and flight mechanics. Wiley New York, 1995, vol. 2.

[29] E.-C. Corporation., DC motors, speed controls, servo systems: an engineering handbook. Oxford, Eng. ; Elmsford, N. Y.: Pergamon Press, 1972.

[30] M. D. Shuster, "A survey of attitude representations," Navigation, vol. 8, no. 9, pp. 439-517, 1993.

[31] P. H. Zipfel, Modeling and Simulation of Aerospace Vehicle Dynamics. American Institute of Aeronautics and Astronautics, Inc., 2014.

[32] D. Brescianini and R. DAndrea, "Computationally efficient trajectory generation for fully actuated multirotor vehicles," IEEE Transactions on Robotics, 2018.

[33] K. J. Aström and R. M. Murray, Feedback systems: an introduction for scientists and engineers. Princeton University Press, 2010.

[34] C. G. Mayhew, R. G. Sanfelice, and A. R. Teel, "Quaternion-based hybrid control for robust global attitude tracking," IEEE Transactions on Automatic Control, vol. 56, no. 11, pp. 2555-2566, Nov 2011.

[35] N. A. Chaturvedi, A. K. Sanyal, and N. H. McClamroch, "Rigid-body attitude control," IEEE Control Systems, vol. 31, no. 3, pp. 30-51, June 2011.

[36] H. Bang, M.-J. Tahk, and H.-D. Choi, "Large angle attitude control of spacecraft with actuator saturation," Control engineering practice, vol. 11, no. 9, pp. 989-997, 2003.

[37] S. Lupashin, M. Hehn, M. W. Mueller, A. P. Schoellig, M. Sherback, and R. DAndrea, "A platform for aerial robotics research and demonstration: The flying machine arena," Mechatronics, vol. 24, no. 1, pp. 41-54, 2014.

[38] M. Bangura, H. Lim, H. J. Kim, and R. Mahony, "Aerodynamic power control for multirotor aerial vehicles," in IEEE International Conference on Robotics and Automation (ICRA), May 2014, pp. 529-536. 


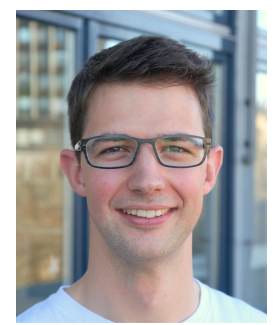

Dario Brescianini received the B.Sc. and the M.Sc. degrees in mechanical engineering from ETH Zurich, Zurich, Switzerland, in 2010 and 2013, respectively. He received the Outstanding D-MAVT Bachelor Award and was awarded the Willi-Studer prize for the best Master's degree in Robotics, Systems, and Control.

$\mathrm{He}$ is currently a doctoral candidate at the Institute for Dynamic Systems and Control, ETH Zurich. His main research interests include aerial vehicle design, the control and trajectory generation of multirotor vehicles, and learning algorithms.

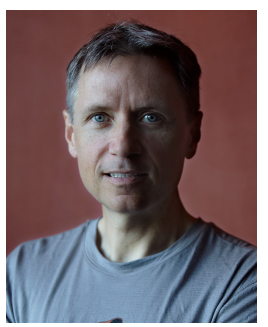

Raffaello D'Andrea received the B.Sc. degree in Engineering Science from the University of Toronto, Toronto, ON, Canada, in 1991, and the M.S. and $\mathrm{Ph} . \mathrm{D}$. degrees in Electrical Engineering from the California Institute of Technology, Pasadena, CA, USA, in 1992 and 1997, respectively.

He was an Assistant and then an Associate Professor with Cornell University, Ithaca, NY, USA, from 1997 to 2007. While on leave from Cornell University, from 2003 to 2007, he cofounded Kiva Systems, North Reading, MA, USA, where he led the systems architecture, robot design, robot navigation and coordination, and control algorithms efforts. He is currently a Professor of Dynamic Systems and Control at ETH Zurich, Zurich, Switzerland, and chairman of the board at Verity Studios AG, Zurich, Switzerland. 